\title{
Expanding services to detect, manage, and prevent pre-eclampsia and eclampsia in Tando Allahyar District of Sindh Province, Pakistan
}

\author{
Ali M. Mir \\ Population Council \\ Irfan Masood \\ Mumraiz Khan \\ Population Council \\ Sharif M.I. Hossain \\ Population Council \\ Tracy McClair \\ Population Council
}

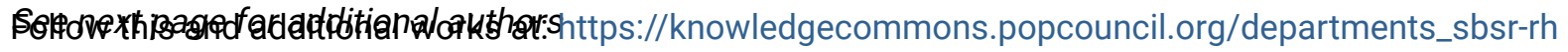

Part of the Demography, Population, and Ecology Commons, Family, Life Course, and Society Commons, Gender and Sexuality Commons, International Public Health Commons, Maternal and Child Health Commons, Medicine and Health Commons, and the Women's Health Commons How does access to this work benefit you? Let us know!

\section{Recommended Citation}

Mir, Ali M., Irfan Masood, Mumraiz Khan, Sharif M.I. Hossain, Tracy McClair, Pooja Sripad, and Charlotte E. Warren. 2019. "Expanding services to detect, manage, and prevent pre-eclampsia and eclampsia in Tando Allahyar District of Sindh Province, Pakistan," Ending Eclampsia Endline Report. Washington, DC:

Population Council. 


\section{Authors}

Ali M. Mir, Irfan Masood, Mumraiz Khan, Sharif M.I. Hossain, Tracy McClair, Pooja Sripad, and Charlotte E. Warren 


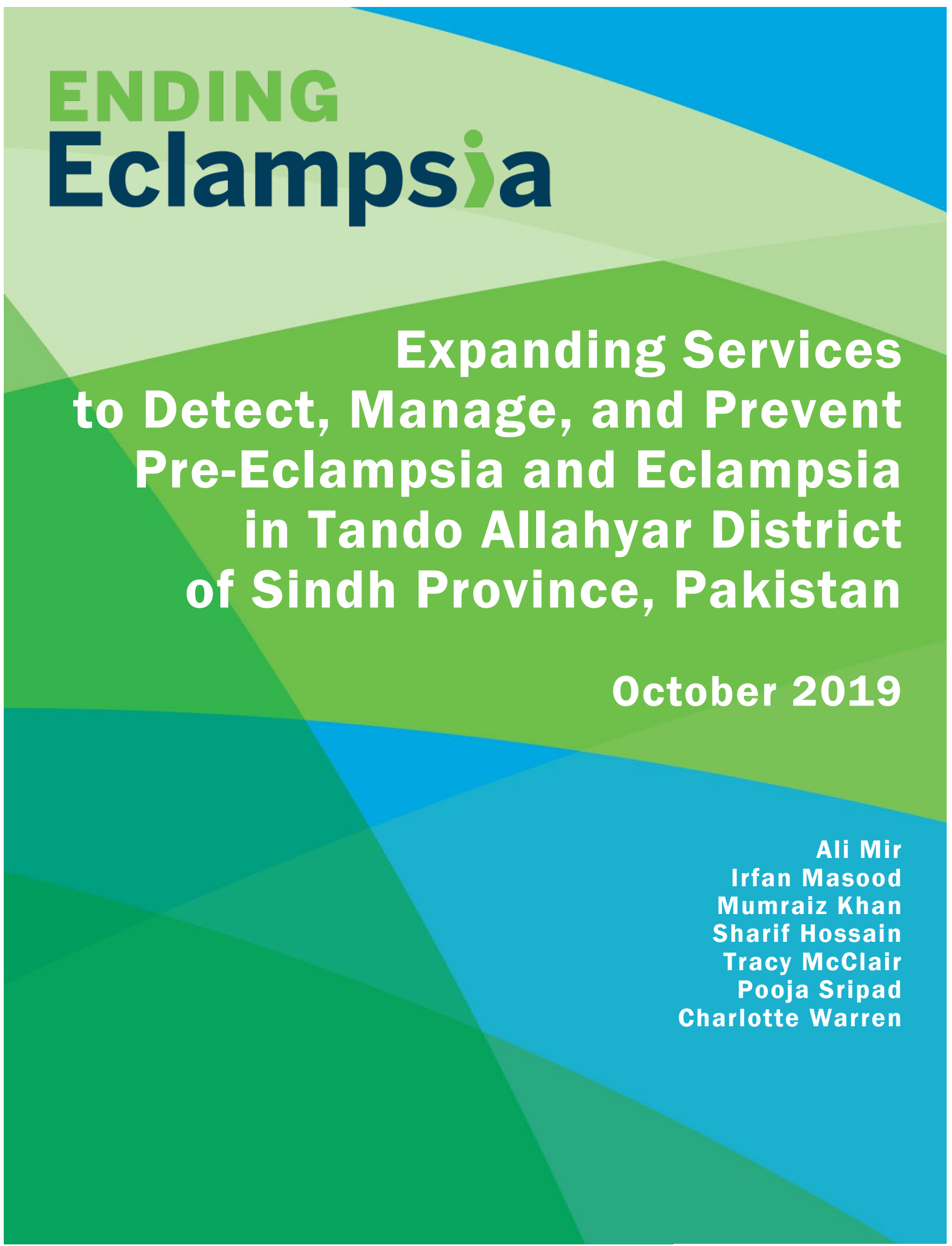




\section{ENDING}

Ending Eclampsia seeks to expand access to proven, underutilized interventions and commodities for the prevention, early detection, and treatment of pre-eclampsia and eclampsia and to strengthen global partnerships.

\section{POPULATION COUNCIL \\ Ideas. Evidence. Impact.}

The Population Council confronts critical health and development issues-from stopping the spread of HIV to improving reproductive health and ensuring that young people lead full and productive lives. Through biomedical, social science, and public health research in 50 countries, we work with our partners to deliver solutions that lead to more effective policies, programs, and technologies that improve lives around the world. Established in 1952 and headquartered in New York, the Council is a non-governmental, non-profit organization governed by an international board of trustees.

\section{Population Council}

4301 Connecticut Avenue NW, Suite 280

Washington DC, 20008

Tel: +1. 877.237.9400

www.popcouncil.org

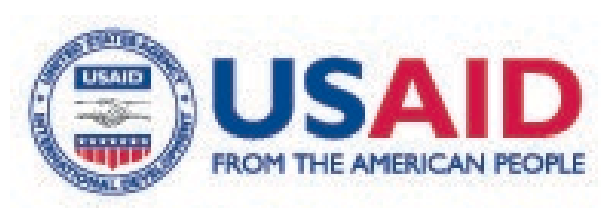

The Ending Eclampsia project is made possible by the generous support of the American people through the United States Agency for International Development (USAID) under the terms of USAID APS-OAA-A-14-00048. The contents of this report are the sole responsibility of the Ending Eclampsia project and the Population Council and do not necessarily reflect the views of USAID or the United States Government.

(c) 2019 The Population Council, Inc. 


\section{Table of Contents}

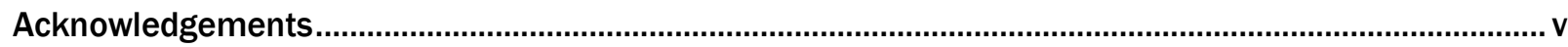

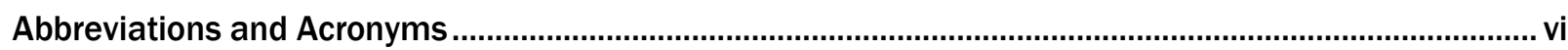

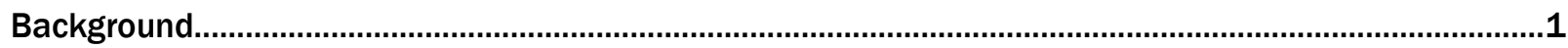

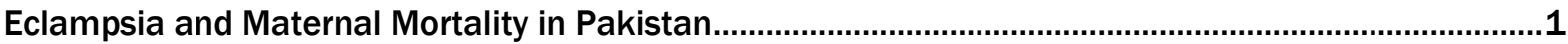

Expanding Services to Detect, Manage and Prevent Pre-Eclampsia and Eclampsia .............................. 3

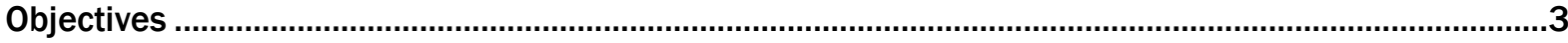

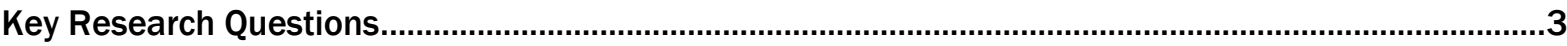

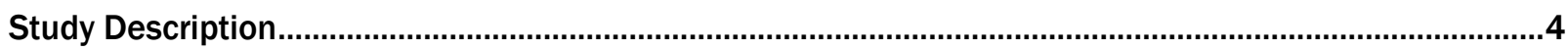

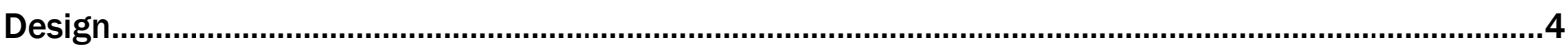

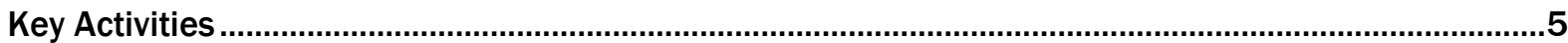

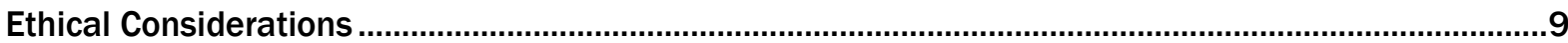

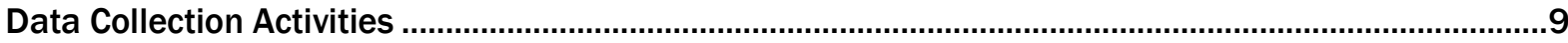

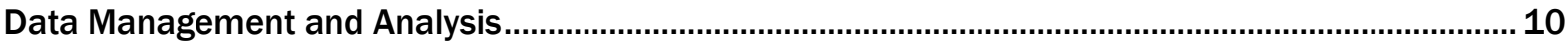

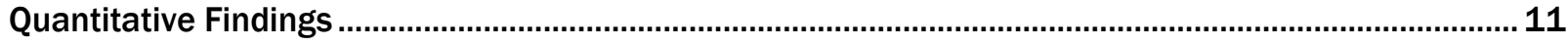

Knowledge and Practice of Community Midwives .............................................................................. 11

Services Provided by Community Midwives Based upon Service Statistical Data .............................. 23

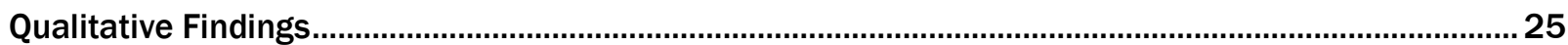

Women's Perceptions of Health Issues in Pregnancy and the Roles of Community Midwives........... 25

Health-seeking Behaviors for Pregnancy Problems.............................................................................. 26

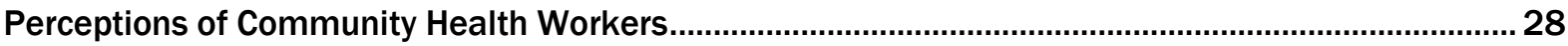

Experiences of Women Managed by Community Midwvies for Pre-Eclampsia or Eclampsia ............ 29

Men's Perceptions of Women's Health Issues During Pregnancy.................................................... 29

Men's Perceptions of Community Midwives and Lady Health Workers .............................................. 31

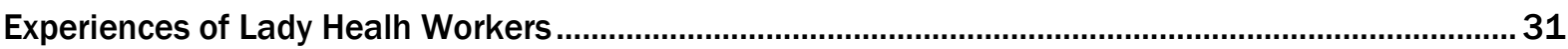

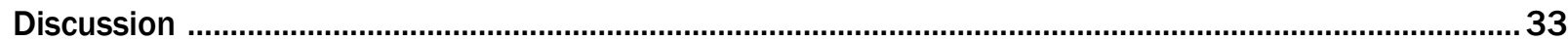

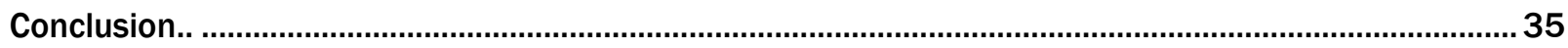

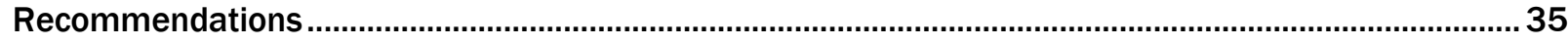

Appendix 1: Availability of Drugs and Contraceptives among Community Midwives ............................. 36

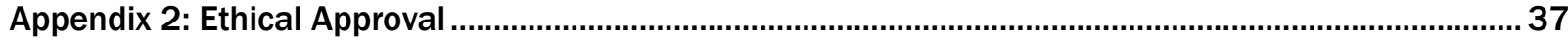

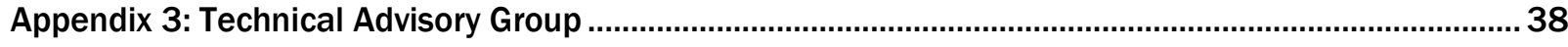

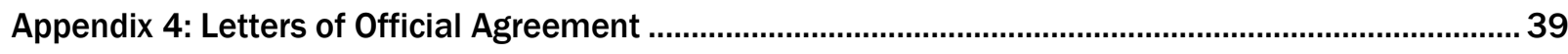




\section{List of Figures}

Figure 1: Four tiers of the public sector health care system in Pakistan .............................................2

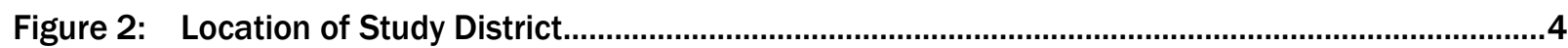

Figure 3: Pictures of eclampsia room established at DHQ Tando Allahyar ..........................................6

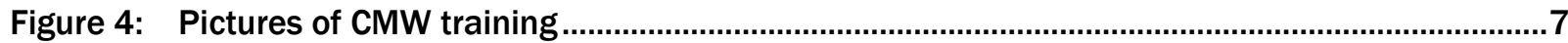

Figure 5: CMWs who correctly identified determinants of maternal morbidity and mortality............. 11

Figure 6: $\quad$ CMW reports of methods for identifying and registering pregnant women ......................... 11

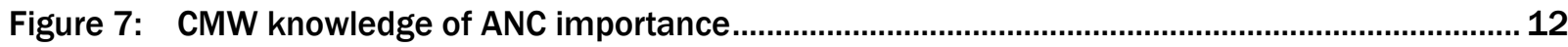

Figure 8: CMW recognition of pregnancy danger signs and symptoms ............................................. 12

Figure 9: CMW knowledge of life-threatening problems during delivery..............................................13

Figure 10: CMW knowledge of postnatal complications .................................................................... 14

Figure 11: CMW knowledge of immediate postnatal assessment ............................................................ 14

Figure 12: CMW knowledge of postnatal counseling topics ................................................................ 15

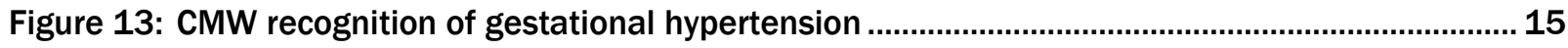

Figure 14: CMW recognition of signs and symptoms of severe pre-eclampsia ....................................... 16

Figure 15: CMW knowledge of maternal risk factors that predispose women to PE/SPE/E................ 17

Figure 16: CMW knowledge of pre-eclampsia management ......................................................... 18

Figure 17: CMWs correctly indentifying case scenarios distinguishing between SPE/E

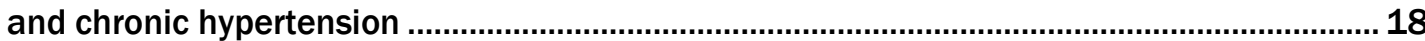

Figure 18: CMW knowledge of severe pre-eclampsia management.................................................. 19

Figure 19: CMW knowledge of eclampsia management ....................................................................... 20

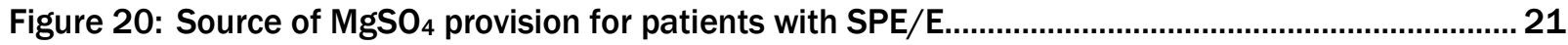

Figure 21: $\mathrm{CWW}$ identification of signs of $\mathrm{MgSO}_{4}$ toxicity and drugs used for treatment...................... 21

Figure 22: CMW reasons for referrals of PE/SPE/E patients.............................................................. 22

Figure 23: CMW opinions of their training and suggestions for improvement ....................................... 22

Figure 24: LHW coordination with CMWs, as reported by CMWs......................................................... 23

Figure 25: ANC, delivery and PNC patients of CMWs during the intervention ......................................... 24

Figure 26: $\mathrm{MgSO}_{4}$ doses by CMWs and private providers during the intervention ................................ 24 


\section{Acknowledgements}

The entire District Health Administration Team of Tando Allahyar played a pivotal role in implementing this Ending Eclampsia project, and their contribution is gratefully acknowledged. Special thanks are owed to Dr. Abdul Quadir Memon, District Health Officer of Tando Allahyar and Dr. Abdul Shakoor Jarwar, District Coordinator of the Maternal, Newborn and Child Health program, for their invaluable support and cooperation.

The National Committee on Maternal and Neonatal Health extended their full support in guiding project implementation, and provided timely recommendations during training material development. Further Committee support from by Professor Sadiqua N. Jafarey and Dr. Azra Ahsan is gratefully acknowledged.

Facilitation provided by Dr. Amna Aziz Memon, Principal of the community midwife school, Mrs. Rubina Gulzar, staff instructor, and other staff members are gratefully acknowledged. During project implementation Dr. Nusrat Nisar, Professor of the Obstetrics and Gynecology department at Bilawal Medical College Jamshoro provided oversight and support. She helped establish the referral links between the community midwives and the referral facility. Professors Nusrat Nisar and Razia Korejo are owed a special mention for their tireless efforts to build the capacities of community midwives to prevent, detect, and manage pre-eclampsia and eclampsia in their communities.

Without the tremendous support and sincere dedication of Dr. Gul Rashida, the successful implementation of project activities would not have been possible. Ms. Amna Yasin, Ms. Shabana, and Ms. Faryal llyas of the Ending Eclampsia team were instrumental in data collection and monitoring activities. Thanks are owed to the global Ending Eclampsia team for their valuable suggestions and guidance at every step of project implementation, especially Dr. Charlotte Warren, Dr. Sharif M.I. Hossain, Amy Dempsey, Karen Kirk, Dr. Pooja Sripad, and Tracy McClair. 


\section{Abbreviations and Acronyms}

$\begin{array}{ll}\text { AE } & \text { Adverse Event } \\ \text { ANC } & \text { Antenatal Care } \\ \text { BHU } & \text { Basic Health Unit } \\ \text { BP } & \text { Blood Pressure } \\ \text { BMC } & \text { Bilawal Medical College } \\ \text { CEmONC } & \text { Comprehensive Emergency Obstetric and Newborn Care } \\ \text { CMW } & \text { Community Midwife } \\ \text { CSPro } & \text { Census and Survey Processing System } \\ \text { DHDC } & \text { District Health Development Center } \\ \text { DHQ } & \text { District Headquarters Hospital } \\ \text { DoH } & \text { Department of Health } \\ \text { DHO } & \text { District Health Officer } \\ \text { EML } & \text { Essential Medicines List } \\ \text { FGD } & \text { Focus Group Discussion } \\ \text { FP } & \text { Family Planning } \\ \text { Ob/Gyn } & \text { Ostetrician/Gynecologist } \\ \text { IEC } & \text { Information, Education and Communication } \\ \text { KP } & \text { Khyber Pakhtunkhwa } \\ \text { LHW } & \text { Lady Health Worker } \\ \text { MAP } & \text { Midwifery Association of Pakistan } \\ \text { MCHIP } & \text { Maternal and Child Health Integrated Program } \\ \text { MgSO } & \text { Magnesium Sulphate } \\ \text { MIS } & \text { Management Information System } \\ \text { mmHg } & \text { Millimeter of Mercury } \\ \text { MMR } & \text { Maternal Mortality Ratio } \\ \text { MNCH } & \text { Maternal, Newborn and Child Health } \\ \text { NCMNH } & \text { National Committee on Maternal and Neonatal Health } \\ \text { NGO } & \text { Non-Governmental Organization } \\ \text { NMR } & \text { Neonatal Mortality Ratio } \\ \text { PDHS } & \text { Pakistan Demographic and Health Survey } \\ \text { PE/E } & \text { Pre-Eclampsia/Eclampsia } \\ \text { PE/SPE/E } & \text { Pre-Eclampsia/Severe Pre-Eclampsia/Eclampsia } \\ \text { PHC } & \text { Primary Health Care } \\ \text { PIH } & \text { Pregnancy-induced Hypertension } \\ \text { PNC } & \text { Postnatal Care } \\ \text { PPHI } & \text { People's Primary Healthcare Initiative } \\ \text { RHC } & \text { Rural Health Center } \\ \text { SAE } & \text { Serious Adverse Event } \\ \text { SBA } & \text { Skilled Birth Attendant } \\ \text { SOGP } & \text { Society of Obstetricians \& Gynecologists of Pakistan } \\ \text { SPE/E } & \text { Severe Pre-Eclampsia/Eclampsia } \\ \text { SPSS } & \text { Statistical Package for the Social Sciences } \\ \text { TAG } & \text { Technical Advisory Group } \\ \text { THQ } & \text { Tehsil Headquarters Hospital } \\ \text { USAID } & \text { United States Agency for International Development } \\ \text { WMO } & \text { Woman Medical Officer } \\ \text { WHO } & \text { World Health Organization } \\ & \end{array}$




\section{Background}

Hypertensive disorders during pregnancy (HDPs) are important causes of severe morbidity, long term disability, and death among both mothers and their infants. ${ }^{1,2}$ Globally, HDPs complicate up to 10 percent of pregnancies. ${ }^{3}$ Most deaths of mothers and infants caused by severe pre-eclampsia and eclampsia (SPE/E) can be prevented through timely and effective medical care. ${ }^{4}$ The World Health Organization (WHO) recommends magnesium sulphate $\left(\mathrm{MgSO}_{4}\right)$ as the most effective, safe, and low-cost anticonvulsant for the prevention, control, and management of seizure in SPE/E.

\section{Eclampsia and Maternal Mortality in Pakistan}

Maternal and child health problems continue as leading causes of death and disability in Pakistan, which is among the six countries accounting for more than 50 percent of global maternal deaths. ${ }^{5}$ The Pakistan Demographic and Health Survey (PDHS) 2006-2007 estimated a Maternal Mortality Ratio (MMR) of 276 per 100,000 live births. Despite efforts to reduce preventable maternal and neonatal mortality, according to recent estimates the country's MMR is 178 deaths per 100,000 live births, and neonatal mortality is 42 per 1,000 live births (PDHS 2017-18). These mortality ratios are higher than other countries in the region.

PDHS 2006-2007 reported eclampsia as accounting for more than 12 percent of direct maternal deaths. Studies by the Population Council in 2015 estimated the MMR of Punjab and Khyber Pakhtunkhwa (KPK) provinces, using an innovative technique that collected data from community-based informant networks. Both studies found that slightly more than one quarter of maternal deaths in Punjab, and slightly less than one fifth of maternal deaths in KPK, had occurred due to pregnancy-induced hypertension (i.e. eclampsia), making it the second largest cause of maternal deaths in both provinces. ${ }^{6}$

A landscape analysis of SPE/E management in Pakistan showed that MgSO4 appears on every province's Essential Medicines List (EML), with guidelines and protocols on its use. In practice, SPE/E management is not occurring at primary health care (PHC) facilities, however, due to a lack of competency-based training and confidence in administering $\mathrm{MgSO}_{4}$, as well as lack of knowledge about MgSO4 toxicity management. ${ }^{7}$ There is also a wide difference in the levels of knowledge between doctors and paramedics in assessing, detecting, and managing PE/SPE/E, with doctors evincing much better knowledge. ${ }^{8}$

Many communities in Pakistan rely primarily on the fourth tier of the public health care system-communitybased health workers (see Figure 1, next page, for a health system schematic). In such communities, lives could be saved if health workers were trained to recognize women with SPE/E early, to stabilize their condition and refer them to appropriate facilities. Evidence from Bangladesh shows that it is possible to train community $\mathrm{PHC}$ providers to diagnose $\mathrm{PE} / \mathrm{SPE} / \mathrm{E}$, administer the loading dose of $\mathrm{MgSO}_{4}$, and refer patients to a higher level facility. 9,10

1 Duley L. 2009. The global impact of pre-eclampsia and eclampsia. Seminars in Perinatology 33(3): $130-137$.

2 Steegers EA, P von Dadelszen, JJ Duvekot, R Pijnenborg. 2010. Pre-eclampsia. Lancet 376(9741): 631-644.

3 World Health Organization, Make Every Mother and Child Count. The World Health Report 2005. Geneva: World Health Organization.

4 World Health Organization recommendations for prevention and treatment of pre-eclampsia and eclampsia. 2011, WHO. Geneva.

5 Hogan MC, KJ Foreman, M Naghavi, SY Ahn, M Wang, SM Makela, AD Lopez, R Lozano, CJ Murray. 2010. maternal mortality for 181 countries, 1980-2008: a systematic analysis of progress towards Millennium Development Goal 5. Lancet 375(9726).

6 Mir AM, S Shaikh, M Khan, I Masood. 2015. Using the Community Informant Based (Made-In and Made-For) Methodology for Estimating MMR in Punjab. Islamabad: Population Council. www.popcouncil.org/uploads/pdfs/2015RH_MMR-ReportPunjab.pdf

7 Sheikh S, RN Qureshi, AR Khowaja, R Salam, M Vidler, D Sawchuck, P von Dadelszen, S Zaidi, Z Bhutta, CLIP Working Group. 2016. Health care provider knowledge and routine management of pre-eclampsia in Pakistan. Reproductive Health 13(2): 104.

8 Mir AM, S Shaikh, M Khan, I Masood, KR Kirk, A Dempsey, C Warren. 2016. Landscape Analysis Report on pre-eclampsia and eclampsia in Pakistan. Washington, DC: Population Council.

9 Shamsuddin L et al. 2005. Use of parenteral magnesium sulphate in eclampsia and severe pre-eclampsia cases in a rural set up of Bangladesh. Bangladesh Medical Research Council Bulletin 31.2: 75-82.

10 Hossain S, S Roy, K Sultana, C Warren. 2019. Assessing the effect of a primary health care intervention for improving pre-eclampsia and eclampsia knowledge and practice in Bangladesh. Population Council 
Figure 1: Four Tiers of the Public Sector Health Care Delivery System in Pakistan

\begin{tabular}{|c|l|}
\hline First Tier & $\begin{array}{l}\text { Tertiary care hospitals located in the major cities } \\
\text { offer specialized in-patient care. }\end{array}$ \\
\hline Second Tier & $\begin{array}{l}\text { Secondary health care facilities include Tehsil } \\
\text { Headquarters (THQH) and District Headquarters } \\
\text { (DHQH) hospitals, which provide out-patient, } \\
\text { in-patient, and some specialized care. } \\
\text { Usually there is one THQH in each sub-district or } \\
\text { Tehsil, and one DHQH in every district. }\end{array}$ \\
\hline Third Tier & $\begin{array}{l}\text { PHC facilities include Basic Health Units (BHUs) } \\
\text { and Rural Health Centers (RHCs), which offer } \\
\text { mainly preventive, out-patient, and basic in-patient } \\
\text { care. Most BHUs do not offer 24/7 services, which } \\
\text { RHCs are mandated to do. }\end{array}$ \\
\hline Fourth Tier & $\begin{array}{l}\text { Community-based services focus on health } \\
\text { promotion and preventive services from LHWs } \\
\text { (Lady Health Workers); CMWs (community } \\
\text { midwives) are community skilled birth attendants. }\end{array}$ \\
\hline
\end{tabular}

In 2006, the Government of Pakistan introduced an official health care provider cadre, Community Midwives (CMWs), to improve the coverage of skilled birth attendants (SBAs) in the country. CMWs receive 18 months of competency-based training, and although they are trained and deployed by the government, they are not considered government employees and are expected to charge for their services. CMWs receive a stipend from the government for a limited period, initially for two years, but extended.

CMWs conduct infant deliveries within their communities at health houses established by the government. Their supplies are currently provided by the government. Management of PE/SPE/E is included in CMWs' training curriculum, but many are not practically trained in the use of $\mathrm{MgSO}_{4}$. Sindh and Punjab provinces' maternal neonatal child health care $(\mathrm{MNCH})$ programs have obtained supplies of $\mathrm{MgSO}_{4}$, but except in a few pilot districts in Punjab, the drug has not been distributed to CMWs.

Community-based primary health care is currently provided by Lady Health Workers (LHWs), but they are restricted to health education, referrals to health facilities for antenatal care (ANC), and providing iron and folate tablets. While LHWs do not conduct deliveries, their scope of work complements that of CMWs. A 2014 study by the Council, however, found a "disconnect" between LHWs and newly deployed CMWs. ${ }^{11,} 12$

\footnotetext{
11 Wajid A, A Rashida, AM Mir. 2010. Initial Assessment of Community Midwives in Rural Pakistan. Pakistan Initiative for Mothers and Newborns (PAIMAN). Population Council: Islamabad.

12 Population Council. 2014. Assessing the Potential of Developing Synergies Between Community-based Lady Health Workers and Community Midwives in Pakistan: Findings from a Qualitative Assessment. Population Council: Islamabad.
} 


\section{Expanding Services to Detect, Manage and Prevent Pre-Eclampsia and Eclampsia}

This report documents a USAID-supported implementation research project implemented by the Population Council in one district in Sindh province, as part of the global-in Pakistan, Bangladesh, Nigeria, Kenya, and Ethiopia-Ending Eclampsia initiative. This implementation research project, "Expanding Services to Detect, Manage, and Prevent Pre-Eclampsia/Eclampsia," assesses CMWs' abilities to screen and detect PE/SPE/E in pregnant and postnatal women, and provide a loading dose of $\mathrm{MgSO}_{4}$ to clients suffering from SPE/E, and referrals to facilities for further management. This study also explored opportunities to enhance collaboration between CMWs and LHWs, encouraging LHWs to refer pregnant women for group ANC and postnatal care (PNC) sessions led by local CMWs, for improved ANC and PNC, as well as enhancing CMWs' counseling skills, and enabling their effective management of postnatal hypertension.

\section{Objectives}

The objectives of the project are to:

- Assess the feasibility of using CMWs to prevent, detect, and manage PE/E by providing the loading dose of $\mathrm{MgSO}_{4}$ and referring clients to referral facilities, and

- Assess the feasibility of orienting LHWs to work collaboratively with CMWs and refer pregnant women to CMWs for group ANC and PNC sessions.

\section{Key Research Questions}

The project was designed to answer three key research questions:

- Does training CMWs and LHWs lead to changes in detection, referral, and survival of women experiencing PE/SPE/E?

- Can CMWs detect, manage, and refer women with PE/SPE/E correctly?

- How do group ANC and PNC sessions and strengthened links between LHWs and CMWs influence maternal health behaviors? 


\section{Study Description}

The study was conducted in Tando Allahyar district of Sindh province. The district was selected after consulting with provincial policy-makers, program managers, as well as the donor. District selection involved the following criteria:

- The district should have at least three sub-districts (or tehsils), and

- There should be at least 20 to $30 \mathrm{CMWs}$ deployed in each tehsil.

Figure 2: Location of study district

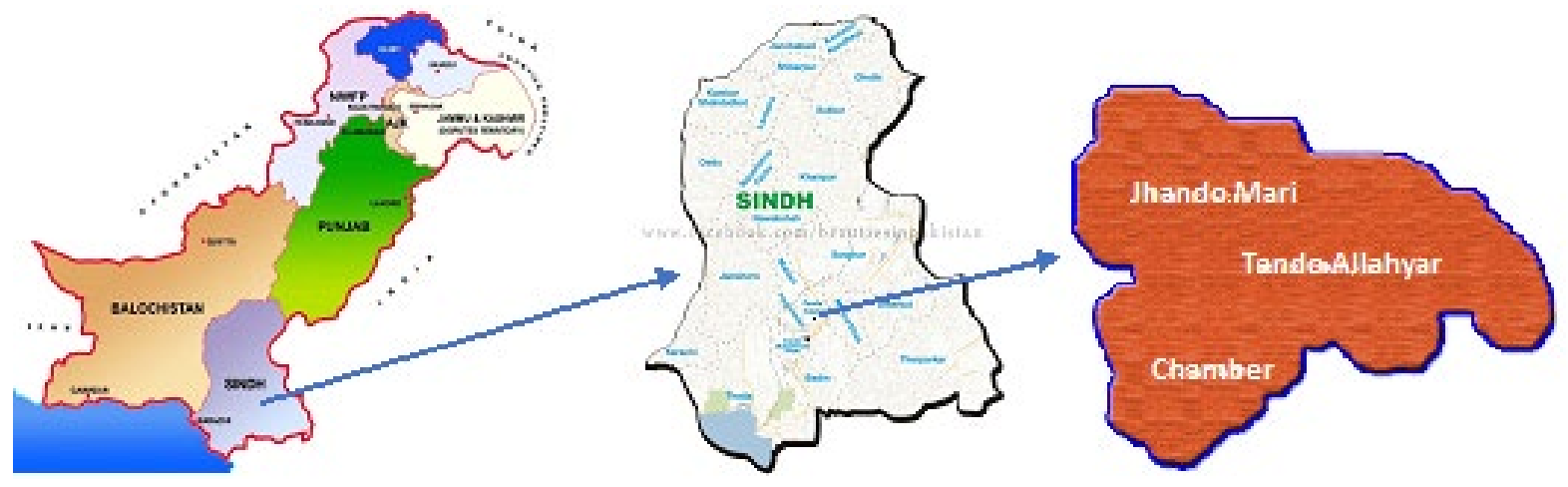

\section{Design}

A quasi-experimental, mixed methods, pre-test, post-test design without any control group was used, which may be expressed as $01 \times 02$, where 01 is the pre-test measurement (baseline), $\mathbf{X}$ is the intervention, and 02 is the post-test measurement (i.e. the results of the endline survey).

The intervention comprised two study arms (summarized in Table 1):

Arm I was implemented in one tehsil, Jhando Mari, in Tando Allahyar district. This project's Primary Healthcare PE/E (PHC PE/E) model of capacity-building and task sharing replicated earlier studies in Nigeria and Bangladesh ${ }^{13}$ examining the feasibility and acceptability of employing CMWs to detect $\mathrm{PE} / \mathrm{SPE} / \mathrm{E}$ cases, administer a loading dose of $\mathrm{MgSO}_{4}$ for SPE/E, and refer clients to appropriate facilities for further management and a maintenance dose. CMWs were trained in individual counseling, specifically conventional ANC and PNC counseling.

Arm II was implemented in other two tehsils in Tando Allahyar district, Tando Allahyar and Chamber. The same PHC-PE/E model in Arm I was implemented, but the Arm II interventions also trained CMWs to conduct group sessions for ANC and PNC, along with orienting and advising LHWs to refer pregnant women to CMWs for group ANC and counseling (on the importance of PNC).

\footnotetext{
13 The studies in Nigeria and Bangladesh, funded by the MacArthur Foundation, were designed to shift the task of providing the loading dose to lower levels of the health system.
} 
Table 1: Activities of the intervention's two study arms

\begin{tabular}{|c|c|c|}
\hline Intervention & Arm I & Arm II \\
\hline $\begin{array}{l}\text { CMW training to identify, prevent, and manage SPE/E prior to administering a } \mathrm{MgSO}_{4} \\
\text { loading dose }\end{array}$ & $\checkmark$ & $\sqrt{ }$ \\
\hline LHW orientation on referring clients to CMWs for ANC and PNC counseling & & $\sqrt{ }$ \\
\hline Individual and conventional ANC and PNC counseling by CMWs & $\sqrt{ }$ & \\
\hline Group ANC and PNC counseling by CMWs & & $\sqrt{ }$ \\
\hline
\end{tabular}

Interventions in both arms were evaluated through a baseline mixed methods assessment in March 2017 and then for the endline assessment in December 2018 and January 2019. Quantitative surveys assessed the differences in CMWs' knowledge, attitudes, and practices of SPE/PE/E detection, prevention, and management before and after the intervention. All trained CMWs in the baseline assessment were included in the endline.

Qualitative data collection included focus group discussions (FGDs) with LHWs at baseline and endline, men and women in intervention communities, in addition to women who received ANC, intrapartum care, and PNC counseling from CMWs. These data explored LHWs' and CMWs' working relationships, community perceptions of $\mathrm{PE} / \mathrm{SPE} / \mathrm{E}$ detection and management, and care-seeking behaviors and the quality of care for women with SPE/E

\section{Key Activities}

The five major inter-related activities of the intervention were: 1) engagement with policy-makers, informing provincial and district program managers about the study, with advocacy for support from the provincial Department of Health (DoH), 2) establishing a provincial pre-eclampsia network, 3) ensuring availability of drugs and supplies, 4) establishing a referral link between communities and referral facilities, and 5) capacity-building for both CMWs and LHWs.

\section{Engagement with Policy-makers}

Meetings informed provincial DoH officials of the study's objectives and sought their support for project implementation, which included ensuring $\mathrm{MgSO}_{4}$ provision to $\mathrm{CMWs}$ in the intervention sub-districts. A District Project Technical Advisory Group (TAG) was constituted with well-defined roles and responsibilities. A district focal person was identified to coordinate intervention activities. Secondary facility staff were trained in $\mathrm{MgSO}_{4}$ administration, to whom trained CMWs referred their SPE/E clients for further management.

\section{Establishing a Provincial Pre-Eclampsia Network}

In December 2017 the process of establishing a pre-eclampsia network started in Sindh province by inviting and engaging policy-makers, members of the Society of Obstetricians \& Gynecologists of Pakistan (SOGP) and Midwifery Association of Pakistan (MAP), and representatives from development partners working on maternal health. Population Council project staff discussed the terms of reference and intervention plans with the president of the National Committee on Maternal and Neonatal Health (NCMNH), with an additional meeting with the President and committee members to discuss the formation of the network. Professor Dr. Sadiqua Jaffrey, Professor Azra Ahsan, and Professor Razia Korejo are network champions who advocated at all relevant forums the need for improving community-based eclampsia management and wider use of $\mathrm{MgSO}_{4}$ at all levels of health facilities. 


\section{Ensuring Availability of Drugs and Supplies}

Sindh's DoH was responsible for procuring, storing, distributing, and ensuring $\mathrm{MgSO}_{4}$ injection supplies for all study sites, in addition to other logistical requirements. $\mathrm{MgSO}_{4}$ was supplied to $\mathrm{CMWs}$ through their nearby public health facilities. CMWs provided $\mathrm{MgSO}_{4}$ injections for free to their clients. CMW supervisors were also oriented on supportive supervision. In addition, health education messages were developed and printed on posters for display at CMWs' birthing stations (where CMWs conduct normal deliveries)

\section{Referral System Establishment and Strengthening}

As in other areas of the country, the extant system at the study sites for client referrals to higher level facilities were either weak or non-existent. Establishing and strengthening referral systems among various community, district, and provincial service providers involved:

1. Introducing CMWs to their community LHWs, who were already providing health education and referrals to the nearest basic health facilities

2. Providing referral slips (developed by Population Council) to each CMW during their training, to enable their referrals of women to proper health facilities

3. Meetings with district health administrators and PHC service providers; service providers shared their phone numbers to facilitate communication among one another

4. Enlisting the Gynecology Department of Liaquat National Hospital and Medical University, Hyderabad to respond to $\mathrm{CHW}$ referral cases, and

5. Strengthening the capacities of the referral facilities; an anesthetist was appointed at DHQ hospital Tando Allahyar.

A functional referral system was successfully initiated through these measures, with the major result of a dedicated eclampsia patient room allocated at DHQ hospital for treatment of eclamptic women for the first time (Figure 3).

\section{Figure 3: Eclampsia room established at DHQ Tando Allahyar}
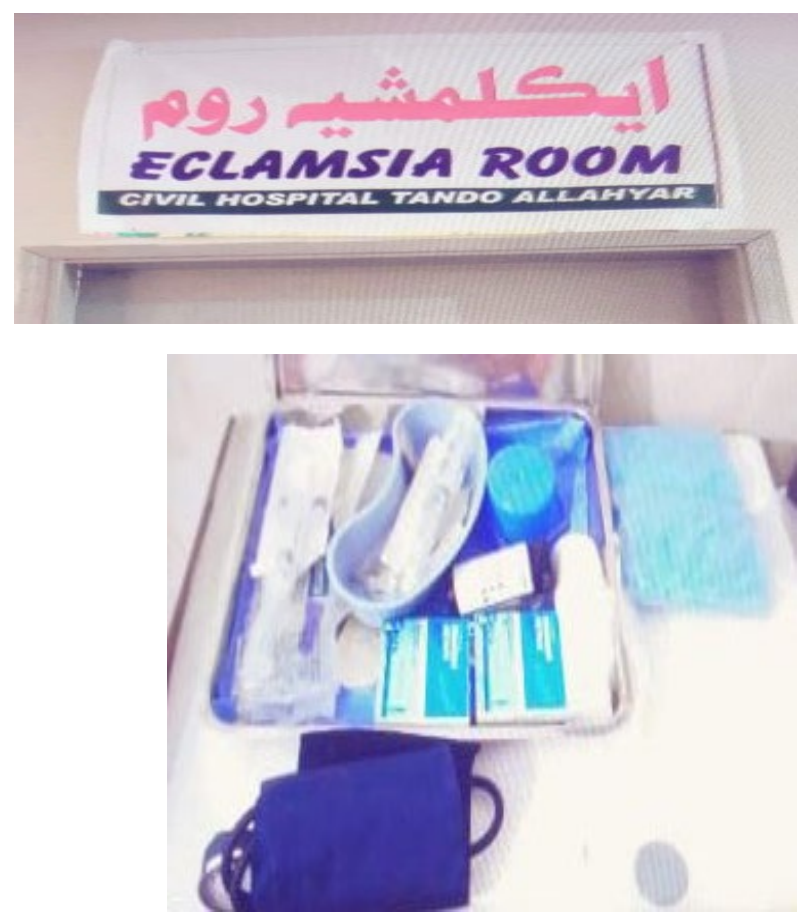
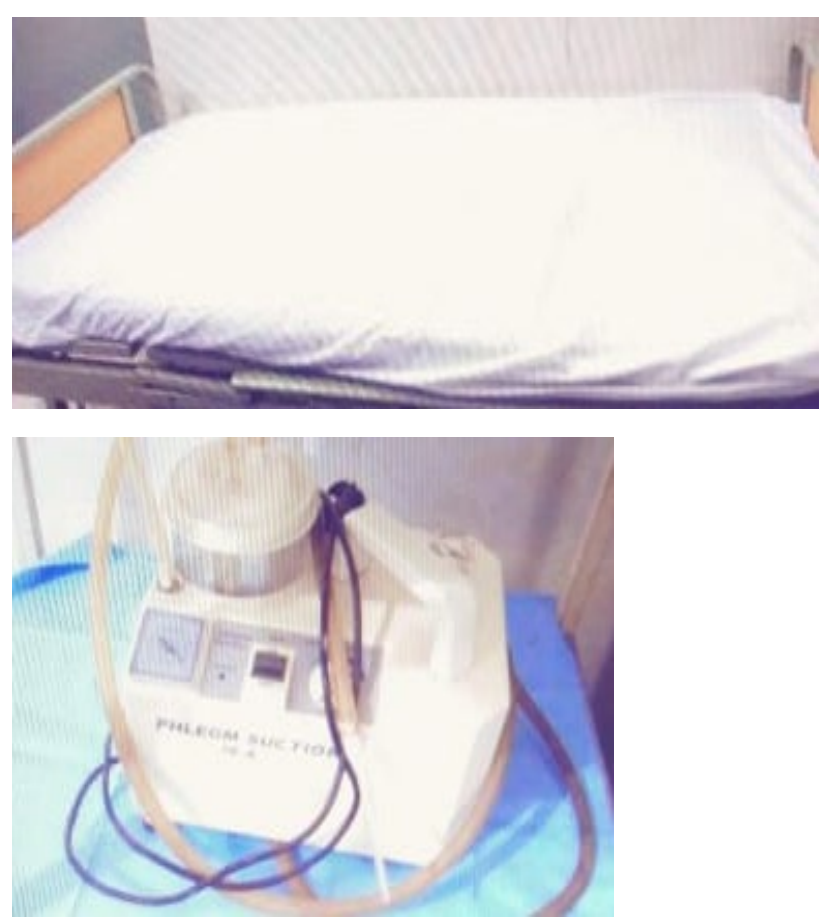


\section{Community Midwife Capacity-building and Lady Health Worker Sensitization}

Two five-day comprehensive CMW trainings were conducted on PE/E screening, detection, prevention, and management at the CMW school in Hyderabad, from 15 to 26 May 2017 (Figure 4). A total of 42 participants including $37 \mathrm{CMWs}$, three Women Medical Officers (WMOs), and two CMW instructors were trained. The trainings were by experienced obstetricians and gynecologists (Ob/Gyns) nominated by the provincial TAG. Participants were provided theoretical and practical knowledge on HDP, definitions of high blood pressure (BP) types during pregnancy, risk factors, $\mathrm{PE} / \mathrm{E}$ danger signs and symptoms, their prevention and management, counseling, referral mechanisms, and the importance of group ANC and record-keeping. Trainees then applied their training in $\mathrm{MgSO}_{4}$ administration. At the end of their training $\mathrm{CMWs}$ were certified by their instructors and NCMNH.

Technical instruction for administering the $\mathrm{MgSO}_{4}$ loading dose employed $\mathrm{MgSO}_{4}$ guidelines developed by the Council, endorsed by the provincial government and now included in the CMW curriculum. The regimen taught was administration of $10 \mathrm{~g} \mathrm{MgSO}_{4}$ injection (5g IM injection in each buttock, $5 \mathrm{~g}$ in $10 \mathrm{ml}$ in $50 \%$ solution) plus $2 \mathrm{ml}$ of one percent Xylocaine intramuscularly. CMWs were trained to be alert to the side effects of $\mathrm{MgSO}_{4}$ and the need for immediate referral of women who had received the loading dose.

Figure 4: CMW trainings
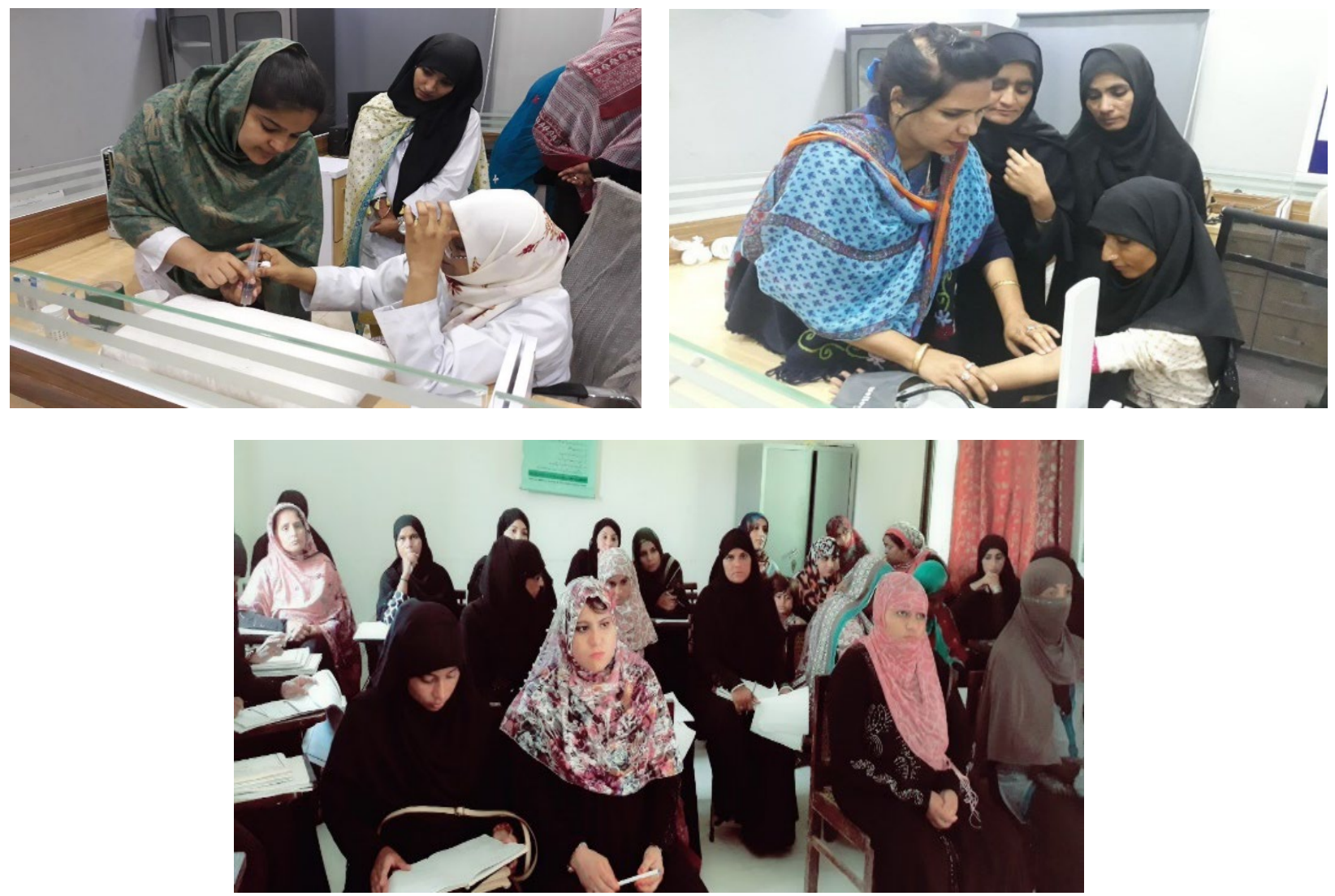

CMWs were also trained to document and report adverse events (AEs) and serious adverse events (SAEs) ${ }^{14}$ associated with $\mathrm{MgSO}_{4}$ administration, using approved forms and reporting procedures. They were trained to note any side effects or toxicity and refer such cases immediately to referral facilities for further management.

${ }_{14} \mathrm{AE} / \mathrm{SAE}$ are defined as any experience suggesting a significant hazard, contraindication, side effect, or precaution associated with use of $\mathrm{MgSO}_{4}$. 
National and provincial guidelines for $\mathrm{MgSO}_{4}$ administration were printed and provided to $\mathrm{CMWs}$. Additional materials developed for a Council safe motherhood project, along with others developed by Jhpiego and $\mathrm{NCMNH}$ on danger signs in pregnancy, were adapted and printed for the CMWs. Information, education and communication (IEC) materials developed specifically for PE/SPE/E were distributed to all CMWs during clinic visits and during monthly meetings, for display at their birthing stations. These IEC materials include pictorial wall charts with easy-to-understand content in the local language.

A one day orientation session for CMW supervisors on supportive supervision enabled their support and guidance for CMWs' loading dose administration. Three clinical supervisors of CMWs from the district's CMW school were also trained, by master trainers, on supportive supervision and monitoring of CMWs. During these trainings, management information system (MIS) tools on key project indicators were introduced and shared with participants. These training activities were carried out in both study arms.

During the second TAG meeting, on 28 December 2017, Sindh's Director General of Health suggested, to increase $\mathrm{MgSO}_{4}$ administration, that a few practitioners in private clinics, in all three sub-districts, should be trained to administer a loading dose of $\mathrm{MgSO}_{4}$ as well, along with strengthening the links between $\mathrm{CMWs}$ and DHQ hospital. Subsequently, from 6 to 9 February 2018, at the CMW school in Tando Allahyar, seven doctors in private practice and 16 paramedics from Jhando Mari sub-district were all trained on $\mathrm{MgSO}_{4}$ protocols.

During the intervention, clients with $\mathrm{PE} / \mathrm{SPE} / \mathrm{E}$ in both study arms who received a loading dose of $\mathrm{MgSO}_{4}$ from CMWs were referred to referral coordinators at the nearest secondary or tertiary care facility. All women diagnosed with PE/SPE/E were closely monitored by CMWs, and any AEs and SAEs observed were reported.

After loading doses of $\mathrm{MgSO}_{4}$ were administered, patients were immediately sent to the referral facility for further management. Clients' addresses and cell phone numbers were recorded for follow up interviews by the referring CMWs. A similar procedure was followed for PNC patients with SPE/E.

In Arm II of the study, LHWs who identify pregnant women and refer them for facility-based delivery were also included in the intervention. Eight sessions, from 19 to 30 July 2017, trained 176 LHWs on PE/E, and registering and referring pregnant women to CMWs for group ANC. The eight sessions were also attended by LHW supervisors and other relevant Basic Health Unit (BHU) staff. These LHW training sessions were conducted by the District Health Development Center (DHDC) trainer. During these sessions, LHWs were introduced to their local CMWs.

During the intervention, women in Arm II who were interested in group ANC were referred to their nearest CMW. Group ANC sessions with pregnant women at similar stages of gestation were held at CMWs' birthing stations or midwifery homes. Eight to 12 women were invited to each group, where they had standard pregnancy health assessments and were provided information, education, and peer support. CMWs informed pregnant women attending the ANC groups of danger signs in pregnancy, preventive care, the importance of BP monitoring, early detection of high BP, as well as other danger signs. CMWs monitored each pregnant woman's BP readings, especially those identified with hypertension or PE/E prior to delivery.

CMWs also counseled their clients on the importance of PNC. Pregnant ANC group attendees were informed that they should each have a PNC visit 1) in the first 48 hours of delivery, 2) after the first seven days, and 3) within six weeks postpartum. 


\section{Ethical Considerations}

Ethical approval was obtained from the Institutional Review Board at the Population Council's headquarters in New York as well as from the National Bioethics Committee of Pakistan.

FGDs and interviews were conducted with service providers as well as with local women and men. Informed consent was obtained from all study participants, after describing, in detail, to them important issues related to the study, and the voluntary nature of any and all participation. After providing their consent for the study, each participant signed a consent form to participate in a discussion. Interviewers described the scope and purpose of the structure questionnaire, its approximate length, and again stressed that participation was completely voluntary.

The structured questionnaire was administered in private. All data collected in every phase of the study were kept confidential and anonymous. Interviews were identified by personal identification numbers rather than participant names.

\section{Data Collection Activities}

\section{Quantitative}

Service statistics were collected at baseline and endline, including the numbers of ANC, delivery, and PNC clients, numbers of hypertensive pregnant clients, and numbers of PE/SPE/E clients-from CMWs, LHWs, and referral facilities.

The baseline and endline interviews of the 37 trained CMWs assessed the trainings' impacts, specifically changes in CMWs' knowledge, attitudes, and practices for PE/SPE/E prevention, detection, and management.

Intervention outcomes were measured by comparing changes between baseline and endline in the number of clients diagnosed with PE/SPE/E in the prenatal period, number of clients administered a loading dose of $\mathrm{MgSO}_{4}$; and number of clients referred to higher level facilities for further SPE/E management.

\section{Qualitative}

Four FGDs with community women who were beneficiaries of CMW services in the three sub-districts were conducted. A total of 47 women ( 21 from Arm I and 26 from Arm II) participated in these discussions, with an average of 12 participants in each FGD.

An additional 12 semi-structured interviews were conducted with women who had received a loading dose of $\mathrm{MgSO}_{4}$ and survived SPE/E, to assess their experiences and overall case management by CMWs and the health system.

Four FGDs with the husbands of women, in all three sub-districts, who received CMW services assessed their knowledge of HDP, its treatment, and their wives' management by CMWs and the health system. All total of 43 men participated in these FGDs with client husbands.

Finally, four FGDs with 53 LHWs-26 from Arm I and 27 from Arm II-assessed the effectiveness of intervention efforts to facilitate collaboration between CMWs and LHWs, as well as improvements in their knowledge of potential pregnancy issues. 


\section{Data Management and Analysis}

Data were processed systematically, with quality controls. The completed questionnaires' first round of quality checks were conducted by the data collection team's supervisor. A member of the Council's research team also performed random checks in the field. Interview sessions were audio-recorded, and discussions were transcribed and stored in MP3 audio files along with respondent fact sheets.

Data were electronically forwarded each day to the data manager, who collated and prepared it for analysis using Microsoft Excel (spreadsheet). The data manager conducted quality checks on questionnaire completeness, and inter-record checks identified mistakes in data files, and suggested corrections in consultation with the principal investigator.

The completed questionnaires were then sent to the Council's office in Islamabad, where they were allotted serial numbers and reviewed again. Any discrepancies identified were referred to the field teams.

Data entry employed CSPro (Census and Survey Processing System) version 7.1.1. After data editing and cleaning, the datasets were checked against original questionnaires for inconsistencies, and corrected if needed. Analysis utilized IBM SPSS Statistics (Statistical Package for the Social Sciences) version 20. Consistency and range checks verified data quality, and original data were recorded to provide data for presentation in tables.

Descriptive means and proportions were used for analysis. Differences between groups were assessed using percentages, and, where appropriate significance tests employed paired t-tests to compare the same respondents at two time points. 


\section{Quantitative Findings}

\section{Knowledge and Practice of Community Midwives}

\section{Determinants of Maternal Morbidity and Mortality and Its Causes}

Compared to the baseline, CMWs' recognition of factors contributing to maternal mortality and morbidity substantially increased. As seen in Figure 5, after training far more CMWs understood that a lack of community awareness of maternal health conditions, transportation issues, for accessing timely care, illiteracy, distances to reach health facilities, and lack of SBAs contribute to adverse maternal health outcomes.

Figure 5: CMWs correctly identifying determinants of maternal morbidity and mortality, $\mathrm{n}=37$

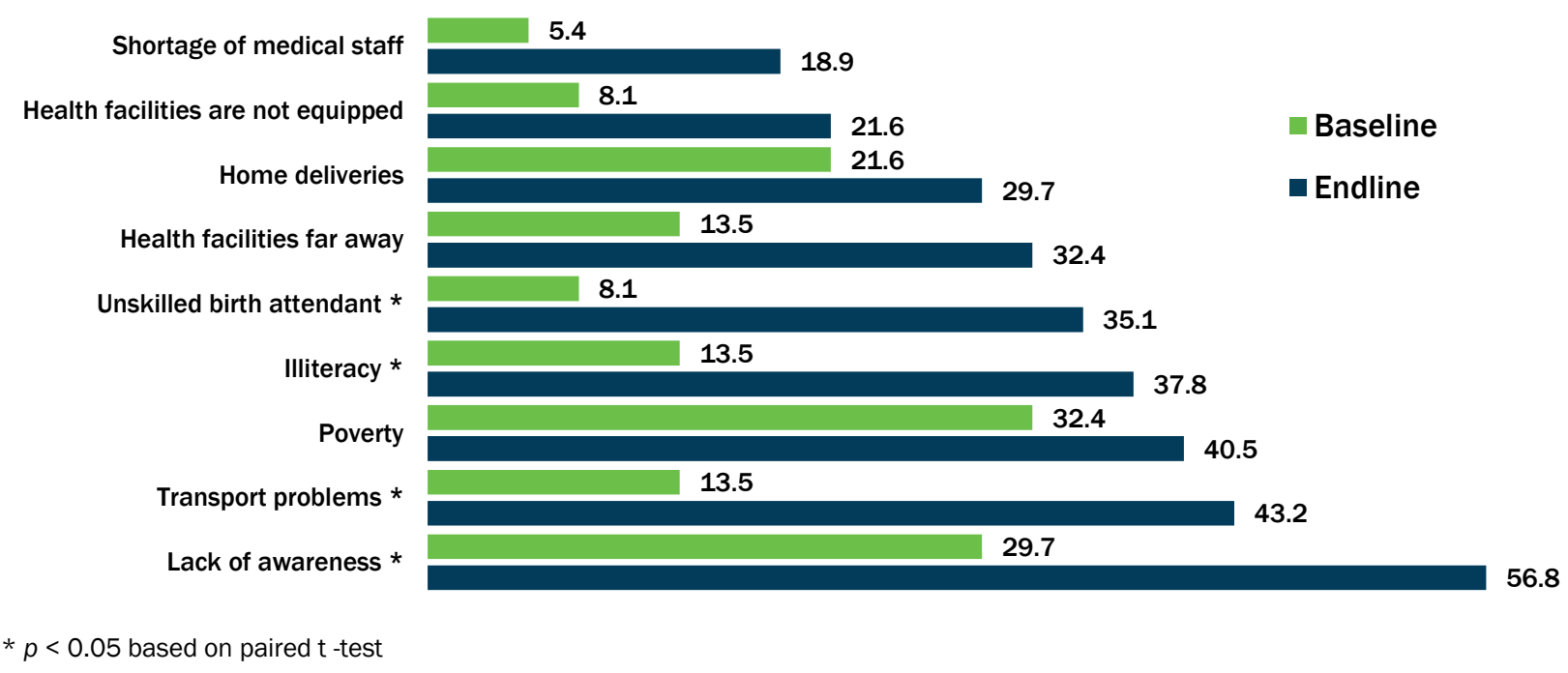

\section{Antenatal Care and Danger Signs}

Identifying and registering pregnant women is the fundamental responsibility of a CMW. More than two fifths of CMWs, at both baseline and endline, that they registered women during home visits This practice remained largely unchanged after CMW training (Figure 6).

Figure 6: CMW reports of methods for identifying and registering pregnant women, $n=37$

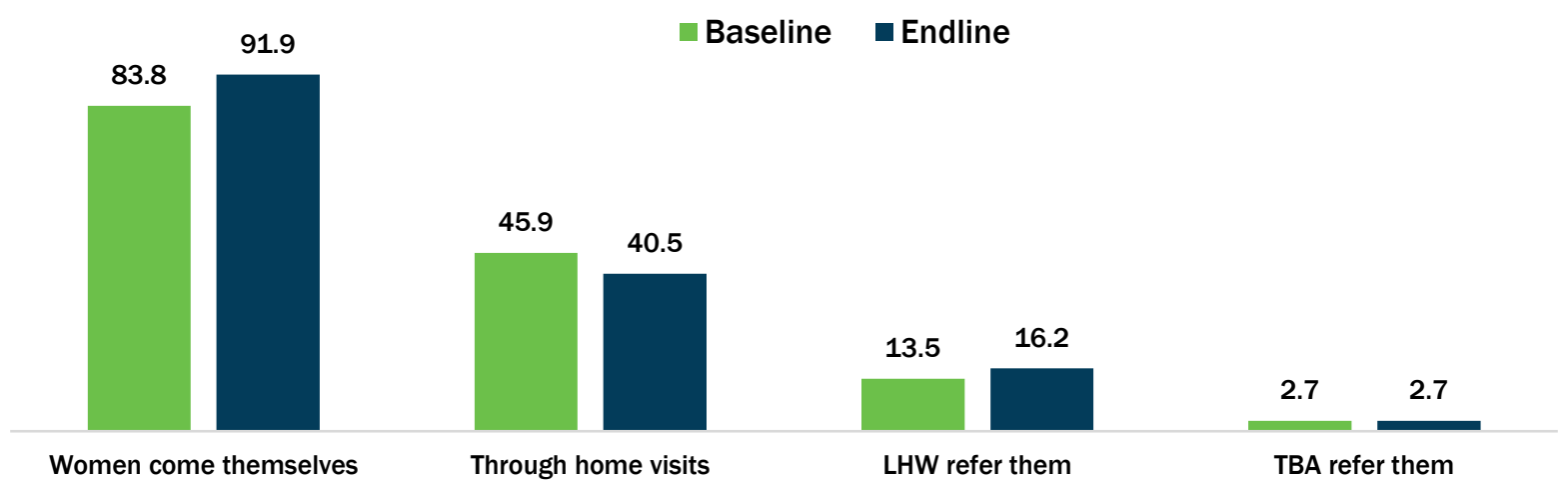


The importance of ANC cannot be overstated, because it aids timely detection of abnormalities and preventable illnesses in both mother and child. Figure 7 shows that CMWs' knowledge improved markedly for the importance of ANC at endline: There were improvements in knowledge of basic concepts and a greater understanding of why ANC is important, especially for avoiding complications and identifying potential problems during delivery and for better planning for child delivery.

Figure 7: CMW knowledge of ANC importance, $n=37$

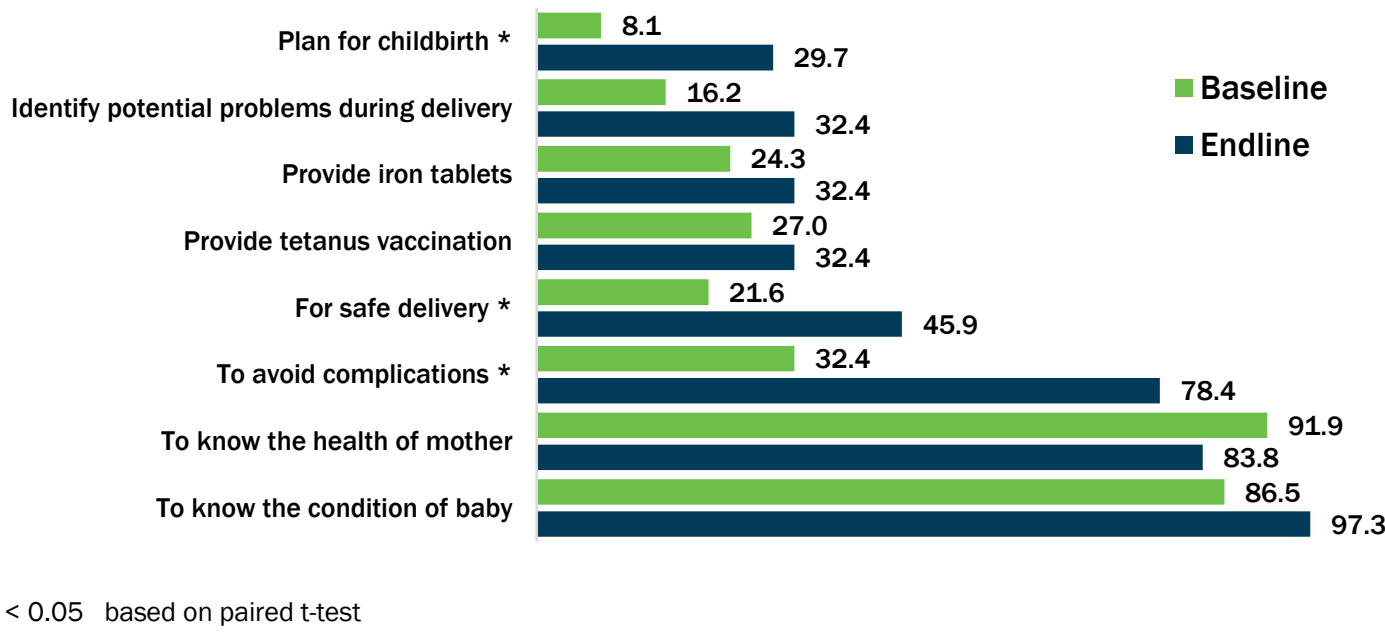

Every CMW must be educated about pregnancy danger signs and symptoms. Complications are often unpredictable, and danger signs indicate potential obstetric complications that can arise during pregnancy, delivery, or after delivery. Knowledge of danger signs can inform CMWs' decisions about appropriate care actions. According to the data in Figure 8, trained CMWs' knowledge of all danger signs and symptoms during pregnancy improved, but for lower abdominal pain, jaundice, persistent severe vomiting, excessive water leaking, foul-smelling vaginal discharge, and proteinuria, it was statistically significant.

Figure 8: CMW knowledge of pregnancy danger signs and symptoms, $n=37$

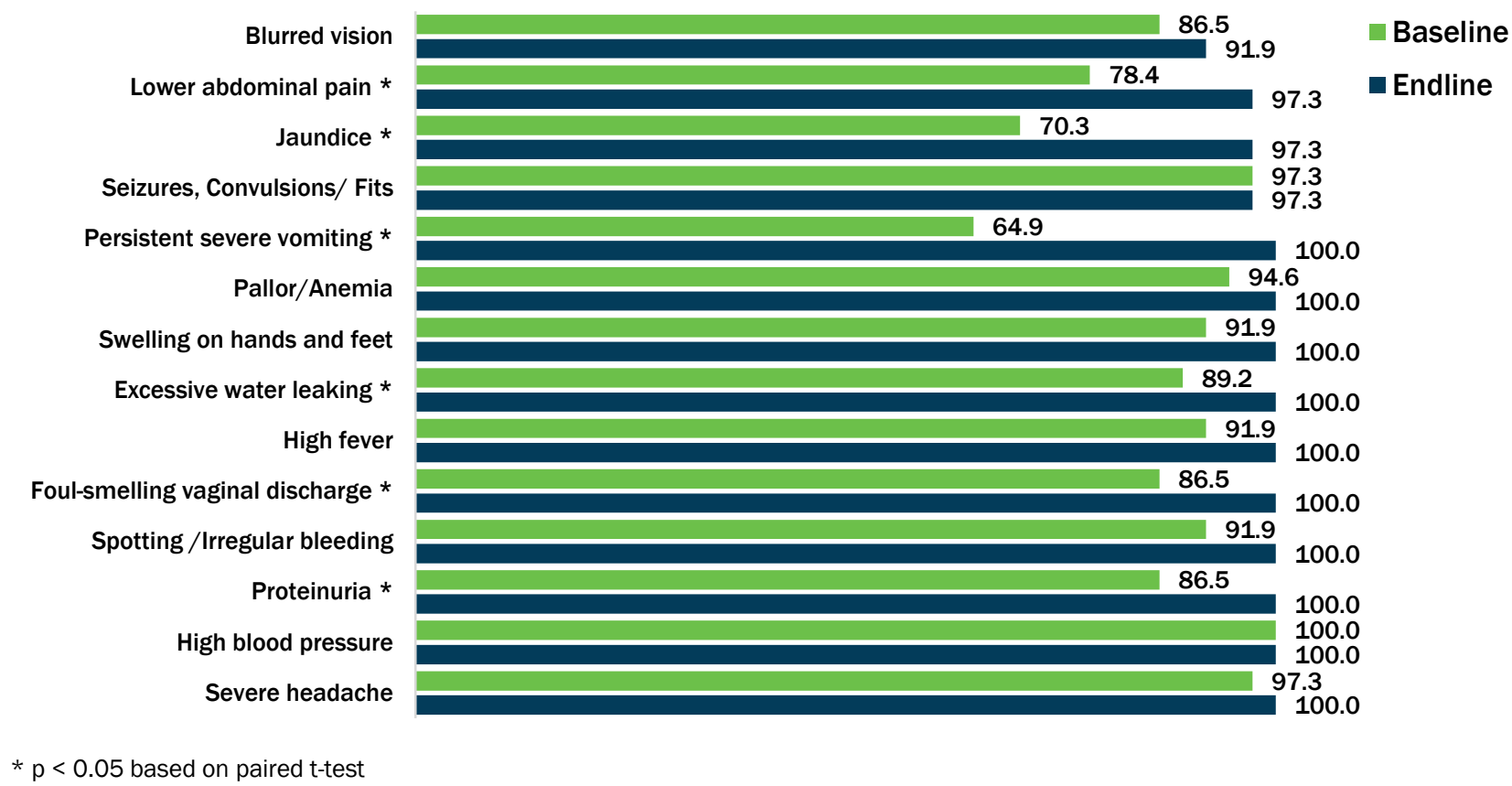




\section{Knowledge of Life-Threatening Problems}

Usually labor and delivery occur without problems-serious problems are relatively rare, with most diagnosed and treated effectively. Sometimes problems develop suddenly and unexpectedly, however. Regular visits to a doctor or certified nurse or midwife during pregnancy can identify problems and improve the chances of a healthy baby and safe delivery. Most CMWs could recognize most pregnancy danger signs before training, but statistically significant improvements were seen after the intervention for recognition of anemia, severe headache, and high fever as possible indicators of life-threatening problems during delivery. Knowledge at baseline was universal for ruptured uterus and heavy excessive vaginal bleeding.

Figure 9: CMW knowledge of life-threatening problems during delivery, $n=37$

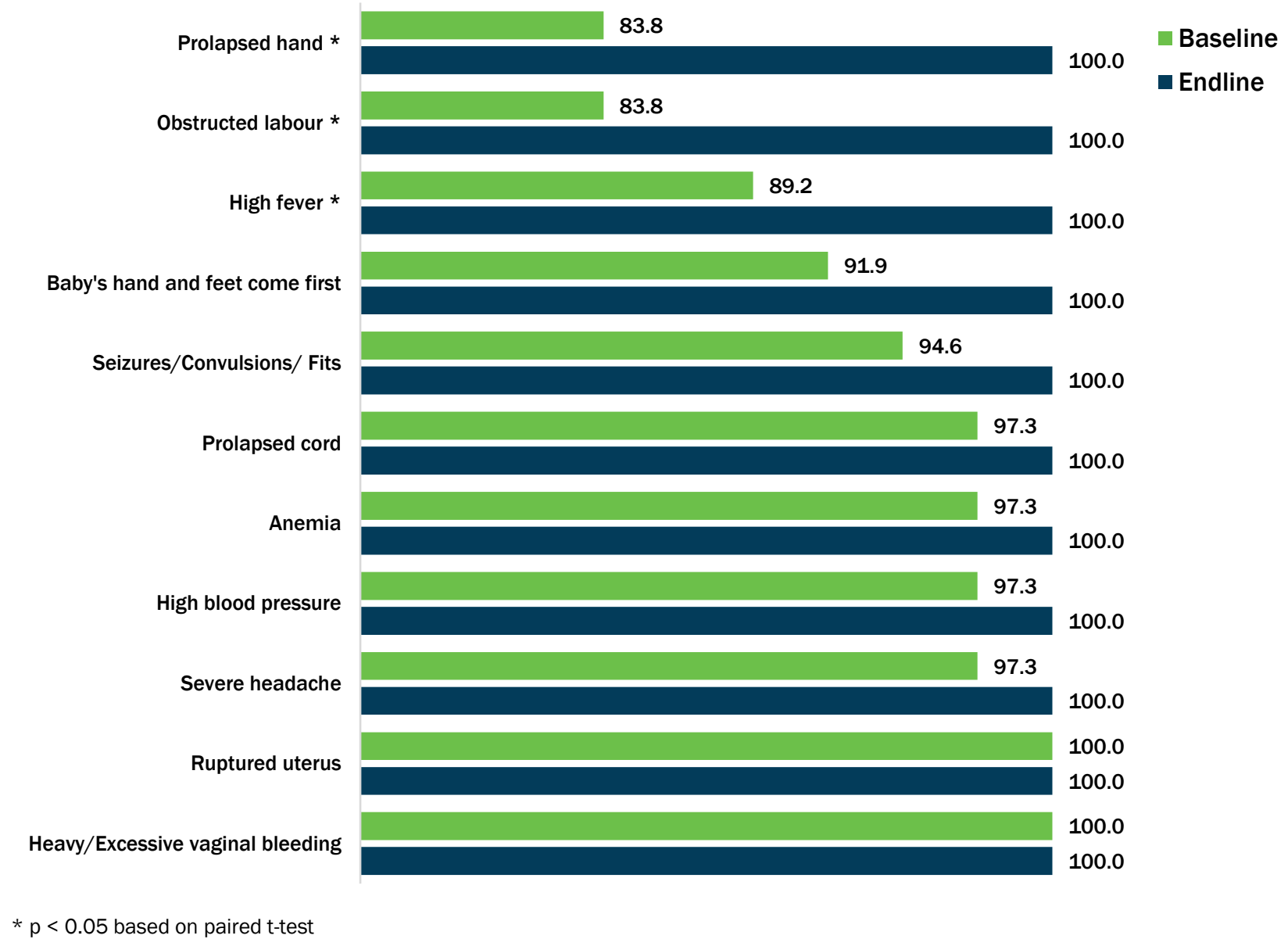

\section{Postpartum Complications and Counseling}

Postnatal care is one of the most important components of health care, helping prevent maternal complications as well as improved maternal and neonatal health. PNC's main purpose is to prevent and diagnose complications early, and encourage breastfeeding and provide family planning (FP) services. Because CMWs provide PNC, they must have good knowledge of postnatal complications. Most CMWs had good knowledge at baseline, which increased notably, particularly for occurrence of high fevers, severe abdominal pain, foul-smelling discharge, shortness of breath or chest pain, and dizziness or palpitations (Figure 10, next page). Postpartum hemorrhage was mentioned as a danger sign by all respondents, at both baseline and endline. 
Figure 10: CMW knowledge of postnatal complications $(n=37)$

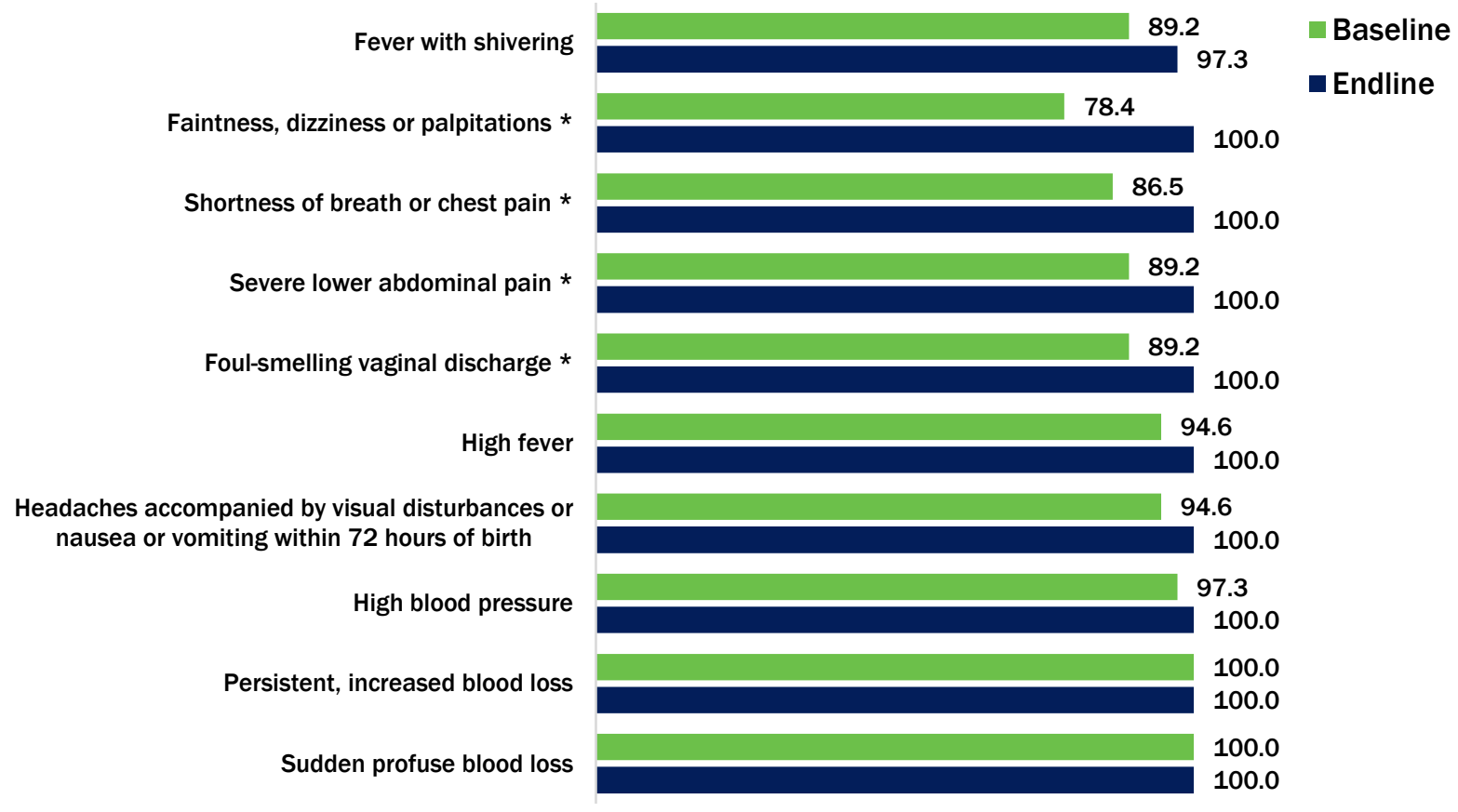

* $p<0.05$ based on paired t-test

CMWs' knowledge of key symptoms and conditions for examination during the immediate postnatal period increased following training, and the changes were statistically significant for increased knowledge of the need to assess perineal wounds, perineal pain, perineal hygiene, and back pain (Figure 11). All CMWs knew, both before and after the intervention, that BP should be checked.

Figure 11: CMW knowledge of immediate postnatal assessment, $n=37$

General and emotional well-being

$$
\text { Fatigue }
$$$$
\begin{aligned}
& \text { Bowel function } \\
& \text { Back pain * }
\end{aligned}
$$

Urinary incontinence

Perineal hygiene *

Perineal pain *

Any perineal wound *

Breast pain

Headache

Vaginal bleeding

Blood pressure

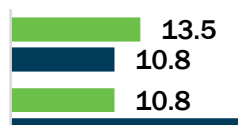

0.8
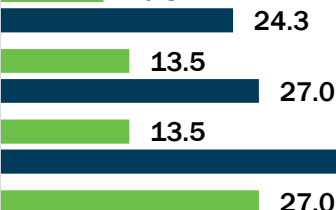

35.1

16.2
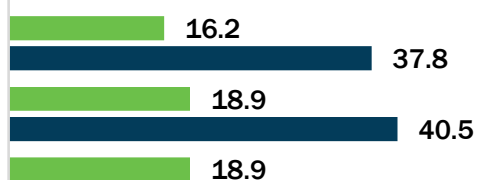

18.9

40.5

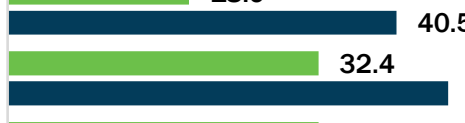

45.9

48.6

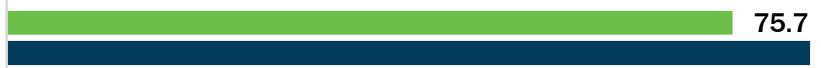

83.8

100.0

100.0

\footnotetext{
$* p<0.05$ based on paired t-test
} 
Figure 12 shows the extent of CMWs' knowledge of PNC counseling topics. After the intervention, CMWs showed enhanced knowledge of counseling on birth spacing, BP monitoring, and immunization. Their knowledge of counseling on postnatal sepsis and nutrition also increased, but the differences were not statistically significant.

Figure 12: CMW knowledge of postnatal counseling topics, $n=37$

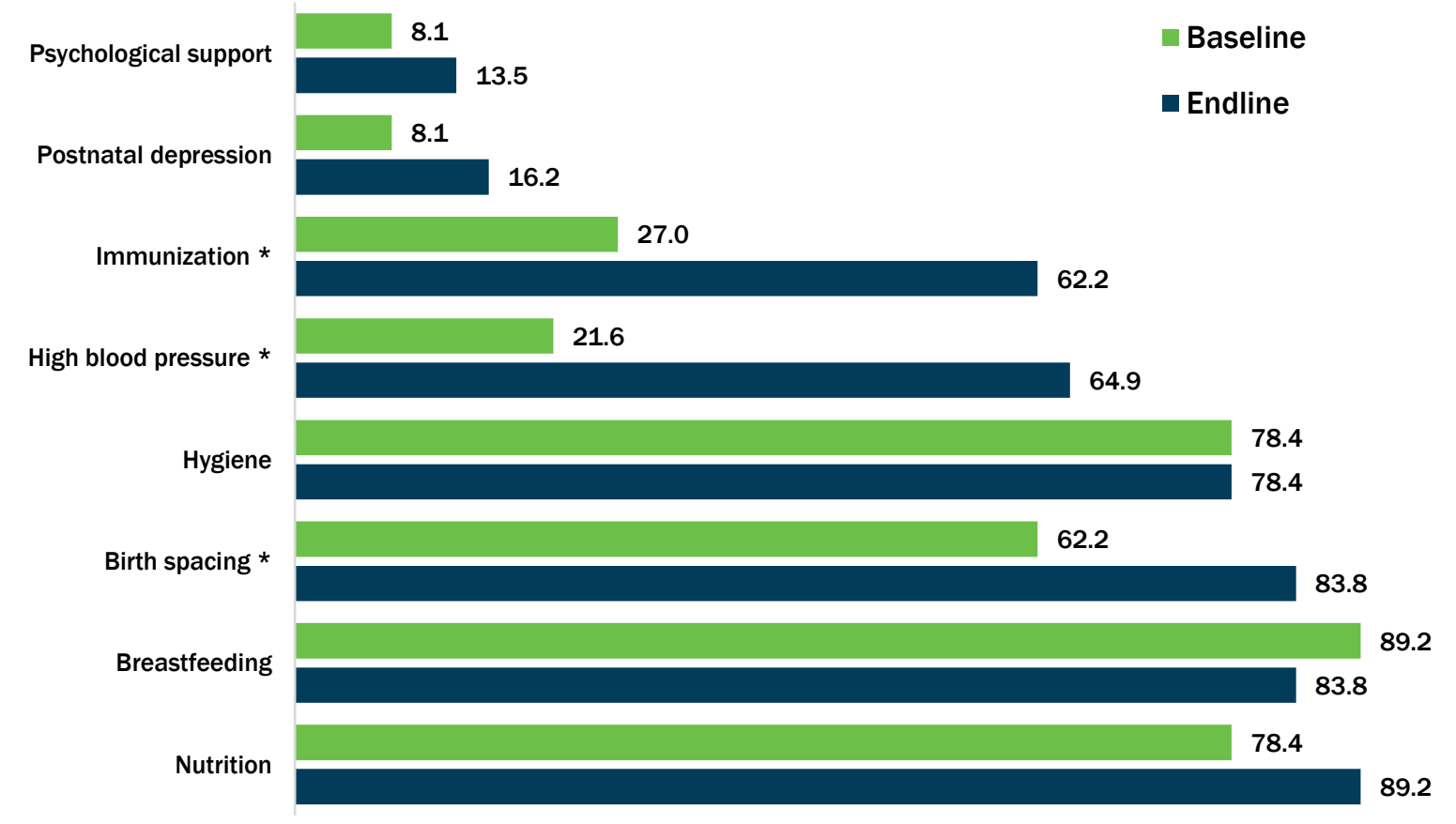

$* p<0.05$ based on paired t-test

\section{Recognition of Hypertensive Disorders of Pregnancy}

Hypertensive disorders of pregnancy are the most common medical complication in pregnancy. As shown in Figure 13, after training, the proportion of CMWs who could accurately recognize gestational hypertension rose by 30 percentage points; this change is statistically significant.

Figure 13: CMW recognition of gestational hypertension, $n=37$

$$
\text { Baseline Endline }
$$

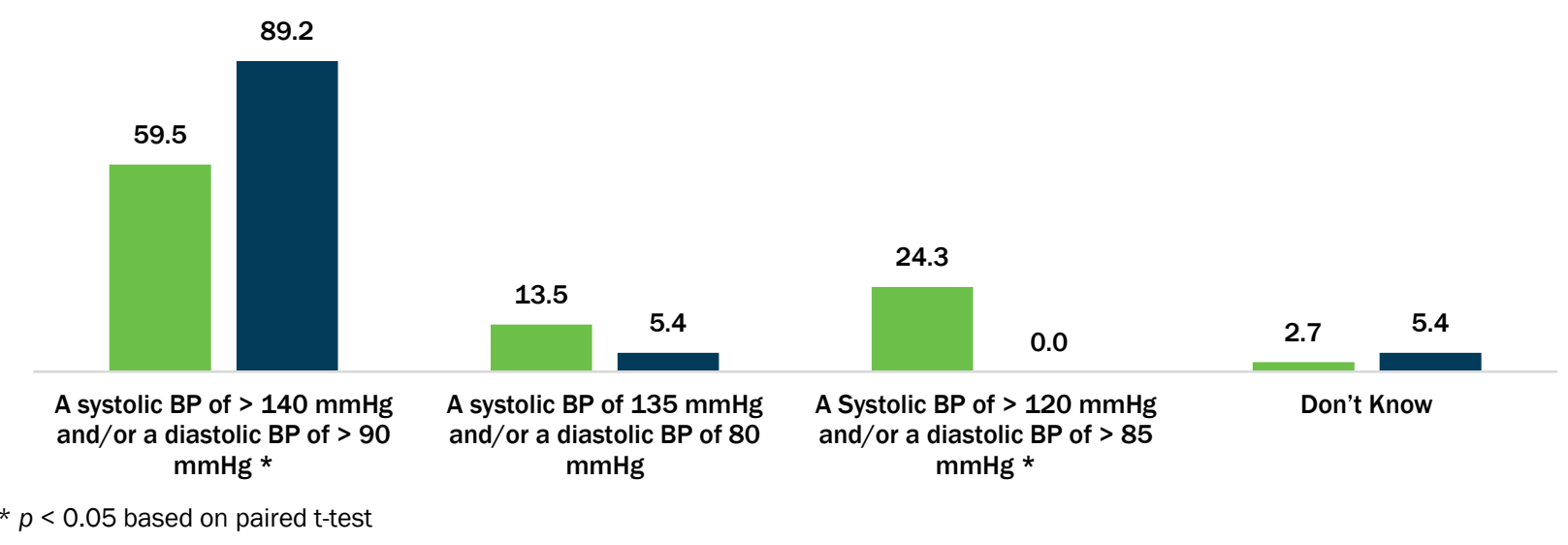




\section{Detecting and Managing Pre-Eclampsia, Severe Pre-Eclampsia and Eclampsia}

Pre-eclampsia rarely happens before the twentieth week of pregnancy. In most cases it occurs before 34 weeks (early onset), and usually towards the end of pregnancy, after 34 weeks (late onset). Although less common, the condition can also develop for the first time in the first six weeks after birth. At endline, 92 percent of CMWs correctly identified systolic BP of $140 \mathrm{mmHg}$ or more and/or diastolic BP of $90 \mathrm{mmHg}$ or more, with excessive protein in urine, as pre-eclampsia. This question was not asked at baseline. At baseline, 14 percent of CMWs identified the importance of ascertaining albumin in urine, while 65 percent did at endline.

A woman is diagnosed with severe pre-eclampsia if she is over 20 weeks pregnant with systolic BP at or above $160 \mathrm{mmHg}$ or diastolic BP at or above $110 \mathrm{mmHg}$, protein in urine 2+ on dipstick, with at least one of the following danger signs: 1) pain in right upper quadrant of abdomen, 2) severe headache, 3) blurred vision, or 4) pulmonary edema. Figure 14 shows a significant increase in CMWs able to recognize these signs and symptoms of severe pre-eclampsia at endline compared to baseline, and the differences are statistically significant for all parameters except high BP.

Figure 14: CMW knowledge of severe pre-eclampsia signs and symptoms, $n=37$

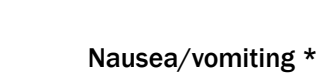

Decreased urine output *

Dizziness *

Blurred vision/light sensitivity/seeing spots *

Swelling (oedema) particularly in face and hands *

Upper abdominal pain (usually under ribs on right side) *

Changes in vision (including temporary loss of vision) *

Severe headaches *

Albumin in urine *

High BP (160/110 or greater after 20 weeks)

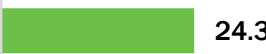

24.3

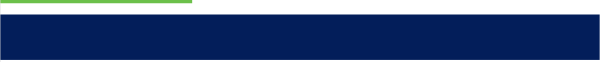

75.7

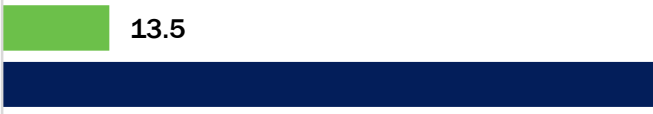

83.8

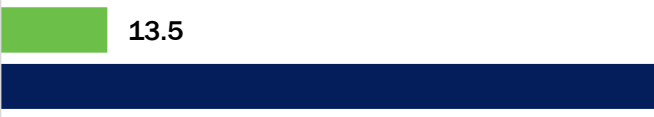

83.8

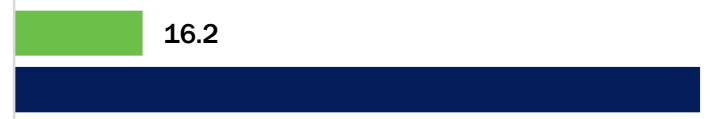

86.5

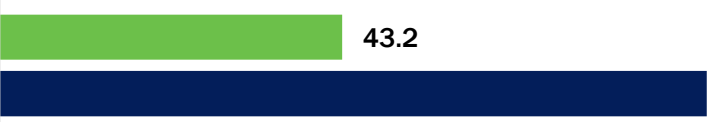

89.2

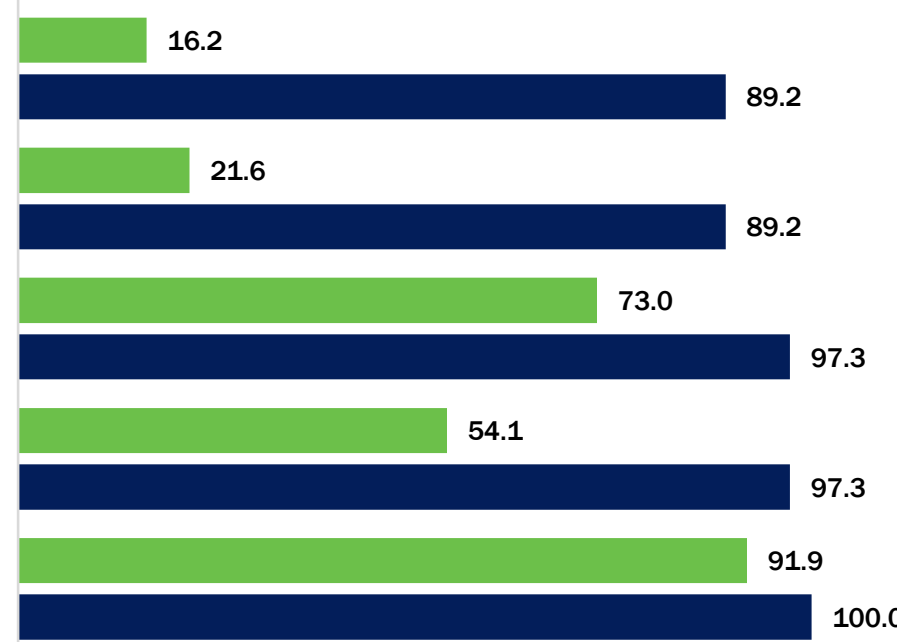

Baseline

- Endline 
CMWs must know the specific maternal risk factors during pregnancy that can lead to PE/SPE/E. Figure 15 shows that, at baseline, CMWs had moderate knowledge of these risk factors. There was a distinct increase in knowledge at endline for all risk factors except chronic hypertension, where the change is around five percentage points, and knowledge of diabetes as a risk factor actually declined. There was a remarkable increase in CMWs identifying first pregnancy, age of 35 years or older, and multiple pregnancies as risk factors; for knowledge of the first pregnancy risk factor, the increase was nearly four-fold from baseline, and statistically significant.

The number of CMWs who could identify family history of pre-eclampsia and chronic kidney disease as risk factors doubled, while those who identified a pregnancy interval of over 10 years as a risk factor tripled, but this was not statistically significant.

Figure 15: CMW knowledge of maternal risk factors predisposing women to PE/SPE/E, $n=37$

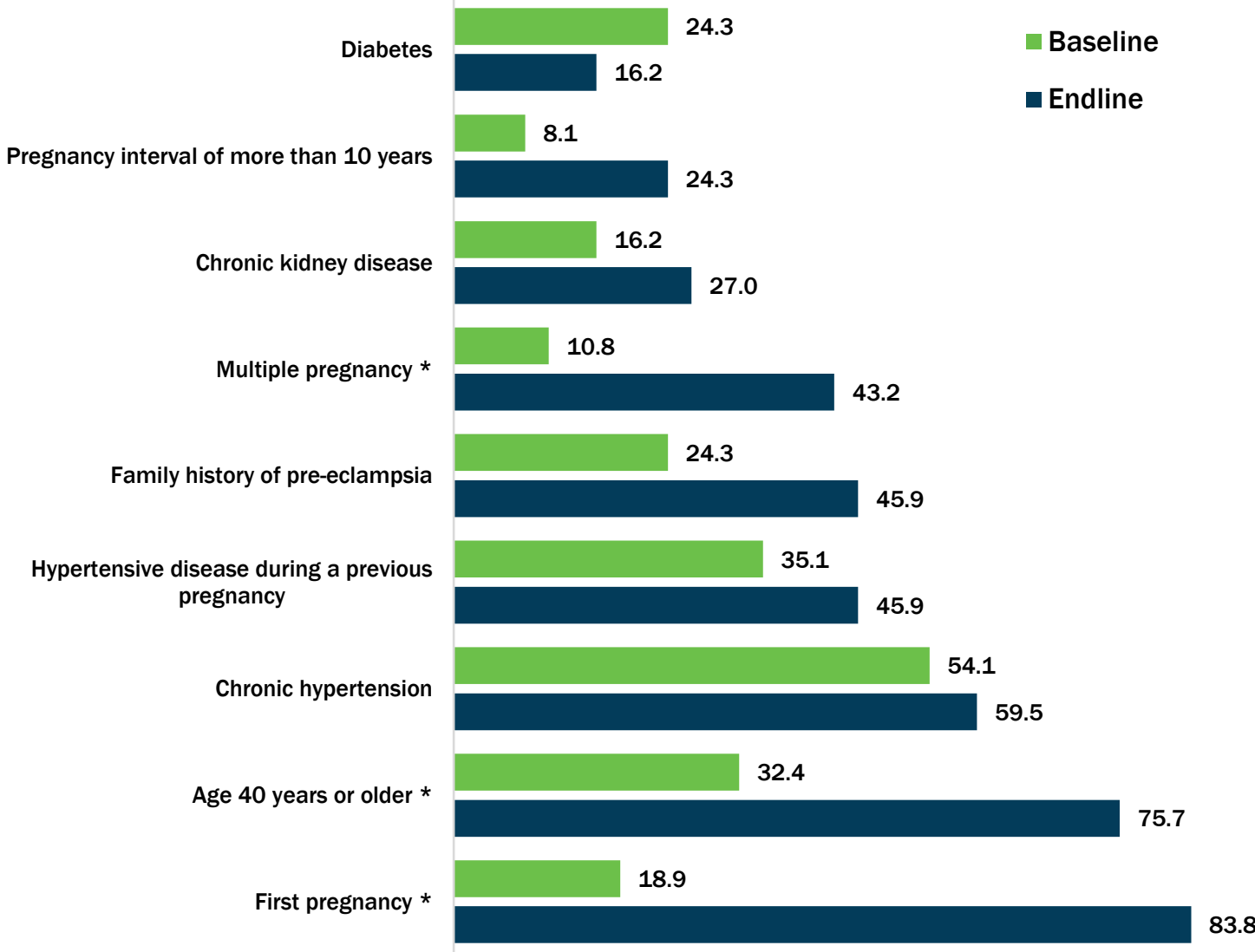

* $p<0.05$ based on paired t-test

When asked about which prophylactic drugs (aspirin and calcium) to prescribe for high risk women, nearly all CMWs (97.3\%) did not know which drugs to use at baseline-approximately half (48.6\%) gave the same response at endline.

At endline, eight percent (data not shown) of CMWs were using aspirin as a prophylactic-it is recommended in their training manual-while none were at baseline. When asked whether they can prescribe aspirin to pregnant women at risk of developing SPE/E, only three CMWs at endline (and none at baseline) stated that they were permitted to prescribe aspirin to prevent pre-eclampsia, which reflects actual stipulations, as CMWs are currently permitted to prescribe aspirin. Only one out of $37 \mathrm{CMWs}$ mentioned calcium supplements as prophylaxis (only recommended for high risk women with low calcium dietary intake). 
According to the results in Figure 16, the most marked change was observed in CMWs' knowledge of preeclampsia management. Knowledge also substantially increased for urine protein checks, antihypertensive administration, advising bed rest, and input and output recordings.

Figure 16: CMW knowledge of pre-eclampsia management, $n=37$

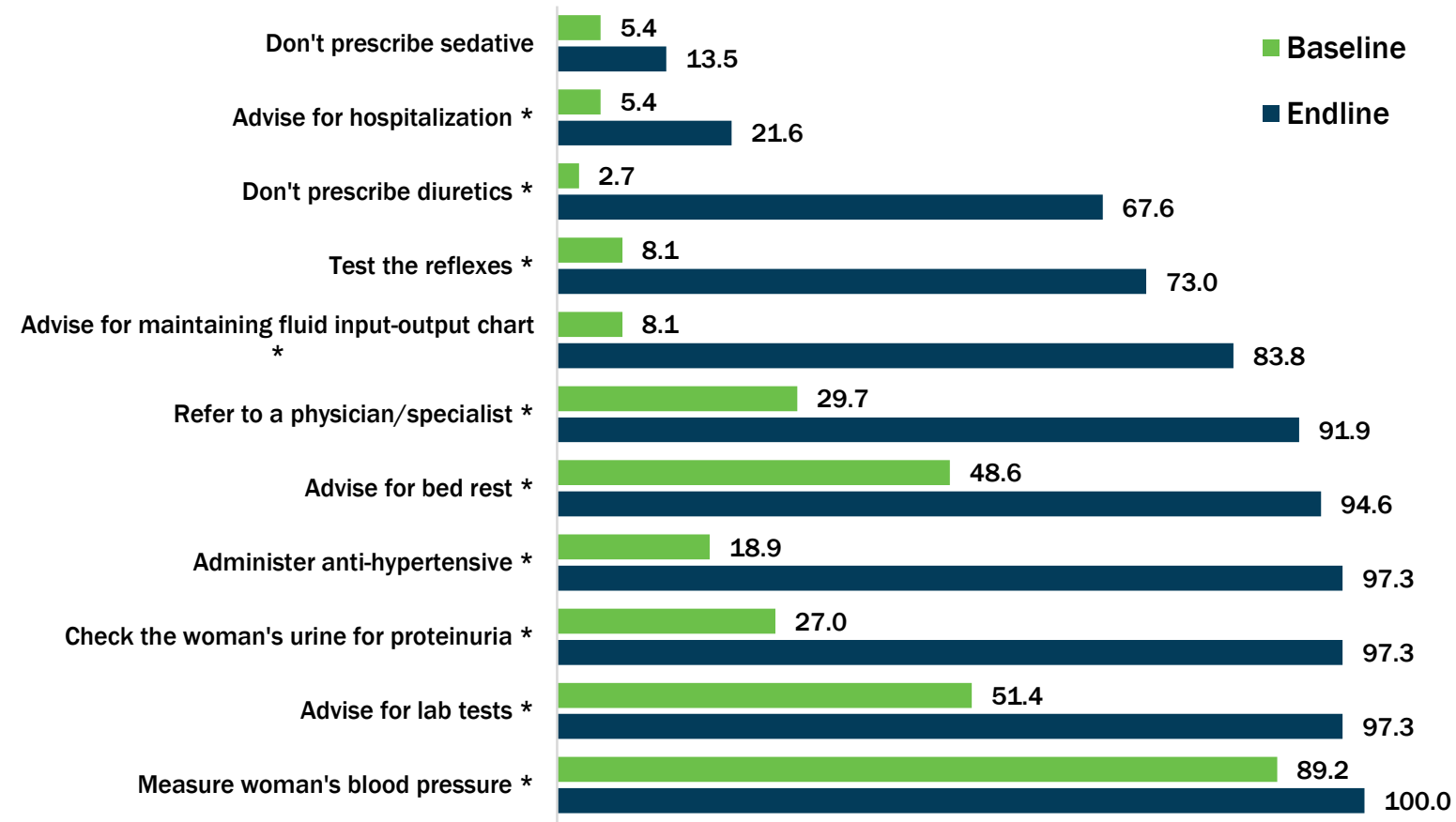

* $p<0.05$ based on paired t-test

To check their abilities to correctly indentify conditions of women suffering from HDPs, CMWs were presented with three scenarios. Figure 17 shows that, compared to baseline, a higher proportion of CMWs at endline were able to diagnose SPE/PE-the differences were statistically significant. At baseline, the greatest proportion-three quarters-of CMWs correctly identified chronic hypertension, and this increased further at endline, by 14 points. Over three fifths of CMWs correctly identified eclampsia at baseline, and this proportion exceeded four fifths at endline.

Figure 17: CMW correct indentification of case scenarios distinguishing between SPE/E and chronic hypertension, $n=37$

Baseline Endline

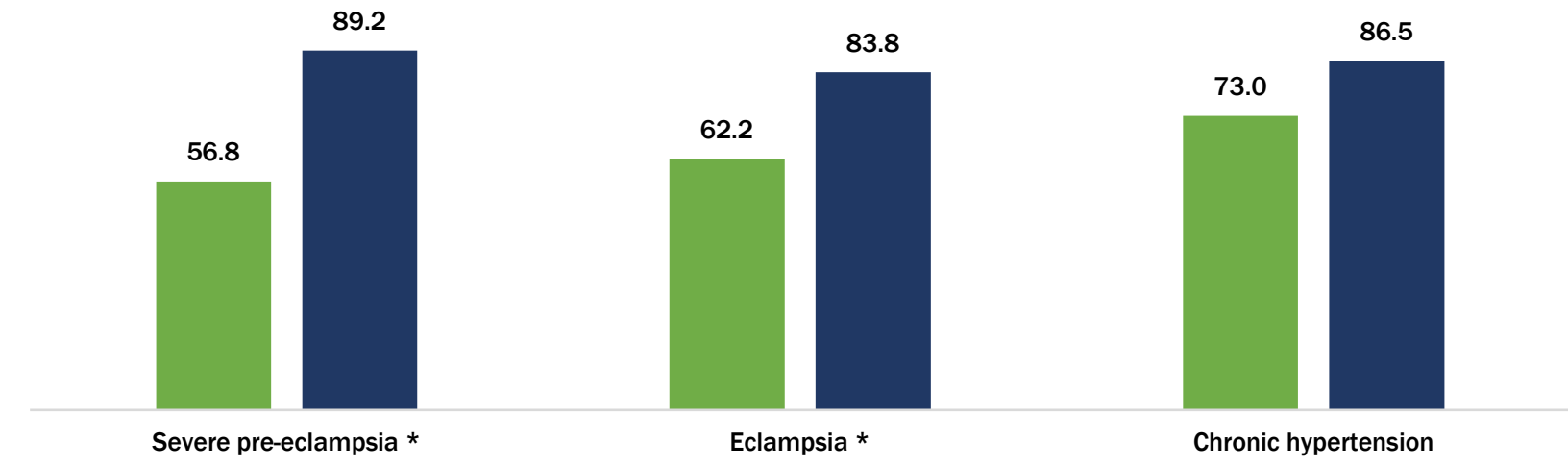

$* p<0.05$ based on paired t-test 
Figure 18 shows substantional improvements in CMWs' responses for correct management of severe preeclampsia. At baseline, one third of CMWs or fewer could identify the measures for managing severe preeclampsia. After the intervention, this knowledge increased to almost universal levels. Improvements in knowledge of all measures were statistically significant, suggesting the training was highly effective.

Figure 18: CMW knowledge of severe pre-eclampsia management, $n=37$

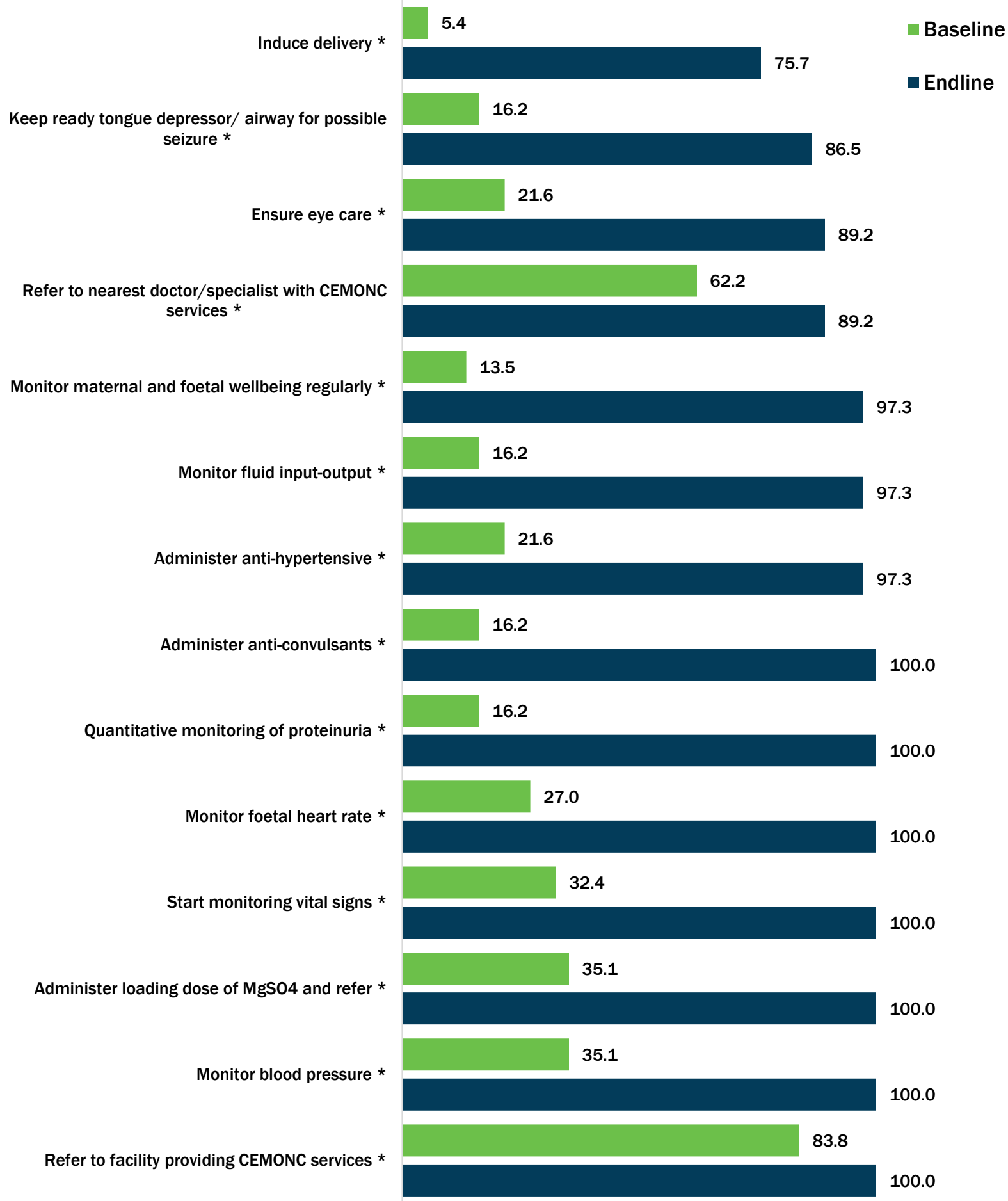

$* p<0.05$ based on paired t-test 
As with severe pre-eclamsia management, there was a marked increase in CMWs' knowledge of eclampsia management (Figure 12). At baseline, only one CMW realized the importance of monitoring fetal heart rate, protein in urine, maternal and fetal well-being, and fluid input and ouput, along with induced delivery. After training, knowledge of these parameters was nearly universal.

Figure 19: CMW knowledge of eclampsia management, $n=37$

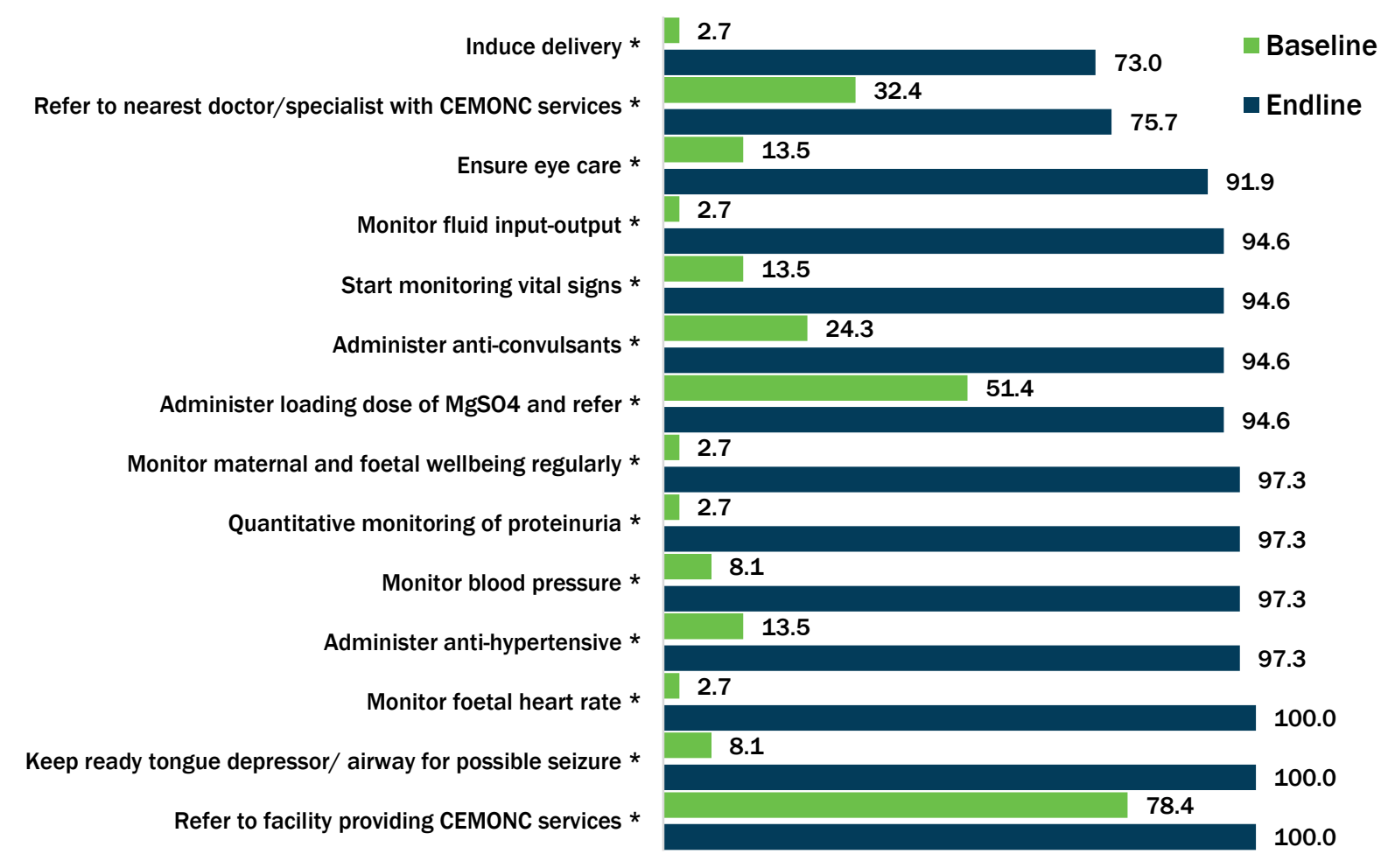

$* p<0.05$ based on paired t-test

\section{Magnesium Sulphate Training and Administration}

Magnesium Sulphate $\left(\mathrm{MgSO}_{4}\right)$, the drug most commonly used as an anti-convulsant for SPE/E management, is usually given by either intramuscular or intravenous routes. WHO recommends $\mathrm{MgSO}_{4} \mathrm{as}$ the most effective, safe, and low cost anti-convulsant treatment for SPE/PE. Four fifths of CMWs at baseline and endline said they had been taught to administer a loading dose of $\mathrm{MgSO}_{4}$ during their basic training. No CMW had subsequent refresher or on-the-job training before the intervention, but 95 percent reported further training with the Ending Eclampsia project. Almost 90 percent of CMWs described their training from the Ending Eclampsia intervention as sufficient.

These results indicate an important achievement of the intervention-an increase in CMWs' confidence in their skills for administering a loading dose of $\mathrm{MgSO}_{4}$. At endline, 84 percent of $\mathrm{CMWs}$ reported sufficient confidence and skills for $\mathrm{MgSO}_{4}$ injection, compared to only 35 percent at baseline-This increase of approximately two and half times is statistically significant. At endline only one fifth of CMWs had recently administered a loading dose of $\mathrm{MgSO}_{4}$, however, likely due to the small number of patients requiring it.

CMWs were asked about the supply of $\mathrm{MgSO}_{4}$ for their facilities. At baseline, more than four fifths of CMWs had no $\mathrm{MgSO}_{4}$ and referred patients to other providers. Slightly over one tenth of CMWs said they were provided the drug by a NGO, while around eight percent bought $\mathrm{MgSO}_{4}$ commercially. Only three percent of CMWs were receiving $\mathrm{MgSO}_{4}$ dosages from district $\mathrm{DoH}$. 
Figure 20 shows that the situation was quite different by endline: Only three percent of CMWs reported not having $\mathrm{MgSO}_{4}$ supplies, while 84 percent were receiving $\mathrm{MgSO}_{4}$ from NGOs, namely Maternal and Child Health Integrated Program (MCHIP), Marie Stopes Society, People's Primary Healthcare Initiative (PPHI), and DKT International Pakistan (Dhanak clinics). Slightly over one tenth were purchasing their doses commercially, and only three percent were supplied by the $\mathrm{MNCH}$ program and $\mathrm{DoH}$.

Figure 20: Sources of $\mathrm{MgSO}_{4}$ for patients with SPE/E, $\mathrm{n}=37$

Baseline Endline

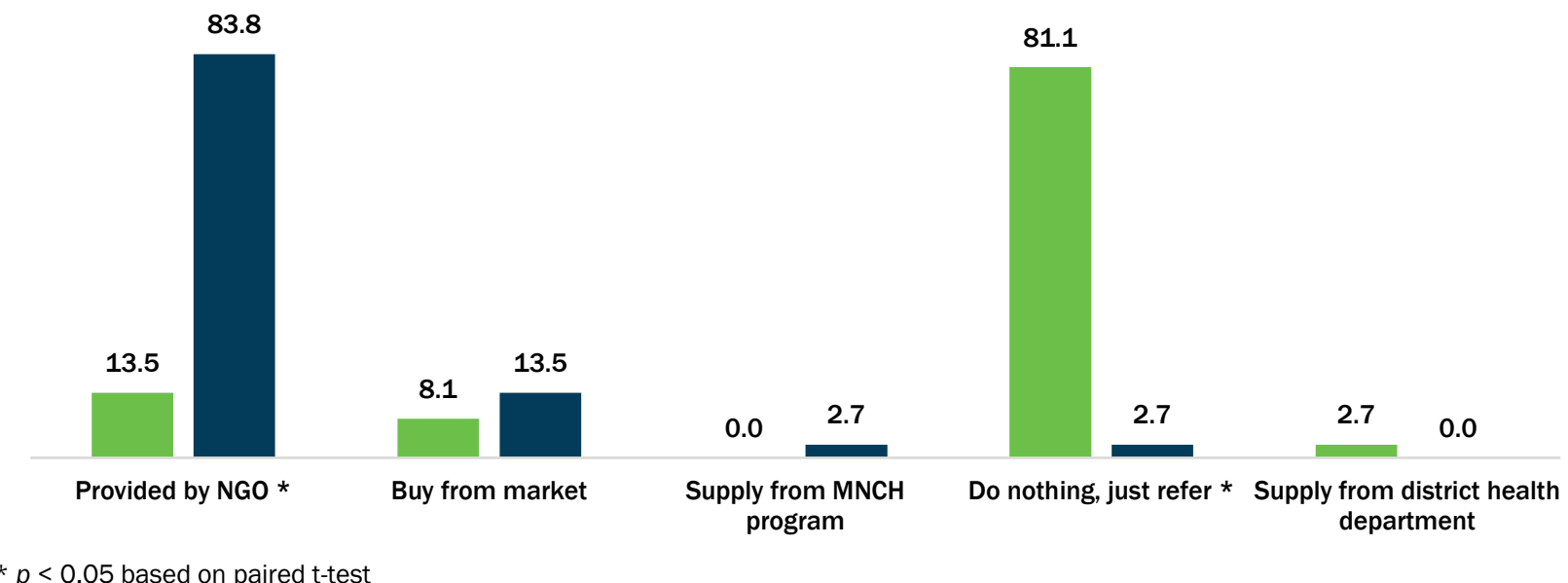

CMWs were asked about their awareness of WHO's recommended Pritchard regime for $\mathrm{MgSO}_{4}$ loading dose administration. Comparison of baseline and endline data indicate that CMWs' knowledge of the regimen was enhanced by 38 percentage points, increasing from 48.6 percent to 86.5 percent.

CMWs were asked whether they had heard of toxicity associated with use of $\mathrm{MgSO}_{4}$. Compared to baseline (13.5\%), a higher proportion of CMWs reported awareness of $\mathrm{MgSO}_{4}$ toxicity at endline (40.5\%). Among CMWs who had heard of toxicity associated with $\mathrm{MgSO}_{4}$, a higher proportion could identify absence of Patella reflexes and decreased urine output as signs of toxicity at endline (Figure 21). There was a reduction in the proportion mentioning respiratory difficulty as a sign, however. The proportion of CMWs who identified calcium gluconate as treatment for $\mathrm{MgSO}_{4}$ toxicity at endline was double that at baseline.

Figure 21: $\mathrm{CMW}$ identification of signs of $\mathrm{MgSO}_{4}$ toxicity and treatment drugs baseline $(n=5)$ and endline $(n=15)$

$\square$ Baseline $\square$ Endline

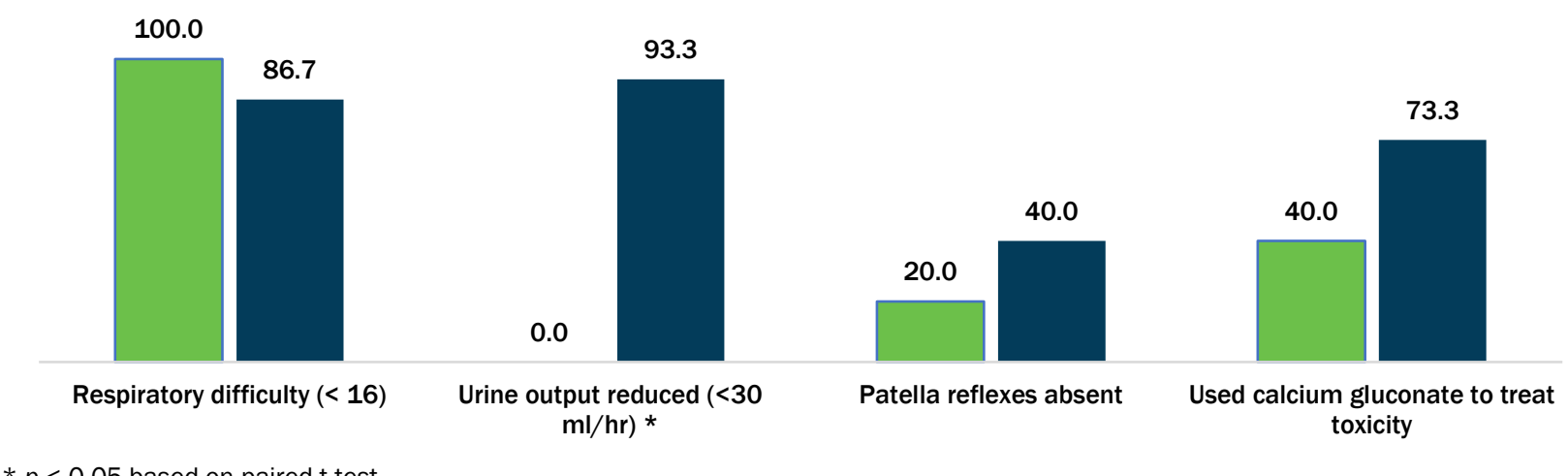

* $p<0.05$ based on paired t-test 


\section{Client Referrals After Magnesium Sulphate Administration}

After administering a loading dose of $\mathrm{MgSO}_{4}, \mathrm{CMWs}$ are required to refer their clients to health facilities for further care. As a result of this requirement, 10.8 percent of $\mathrm{CMWs}$ at baseline reported referring pregnant women for additional medical attention, and at endline more than one quarter (27\%) were. At baseline, CMWs had referred all women to DHQ hospital, but post-intervention, the referral pattern had enlarged and included a teaching hospital and private clinics as well. Providers at these referral facilities included in the referral system developed during the project were trained by the project.

Figure 22 shows that, compared to baseline, at endline CMWs had fewer reasons for referring eclampsia patients to health care facilities. The proportion of CMWs referring such clients due to lack of basic equipment (such as functional BP apratus) had fallen by 30 percentage points, while the proportion of those referring due to inability to manage had reduced by 55 percentage points, and lack medicines was no longer a major reason for referral.

Figure 22: Reasons described by CMWs for referrals of PE/SPE/E patients to facilities baseline $(n=4)$ and endline $(n=10)$

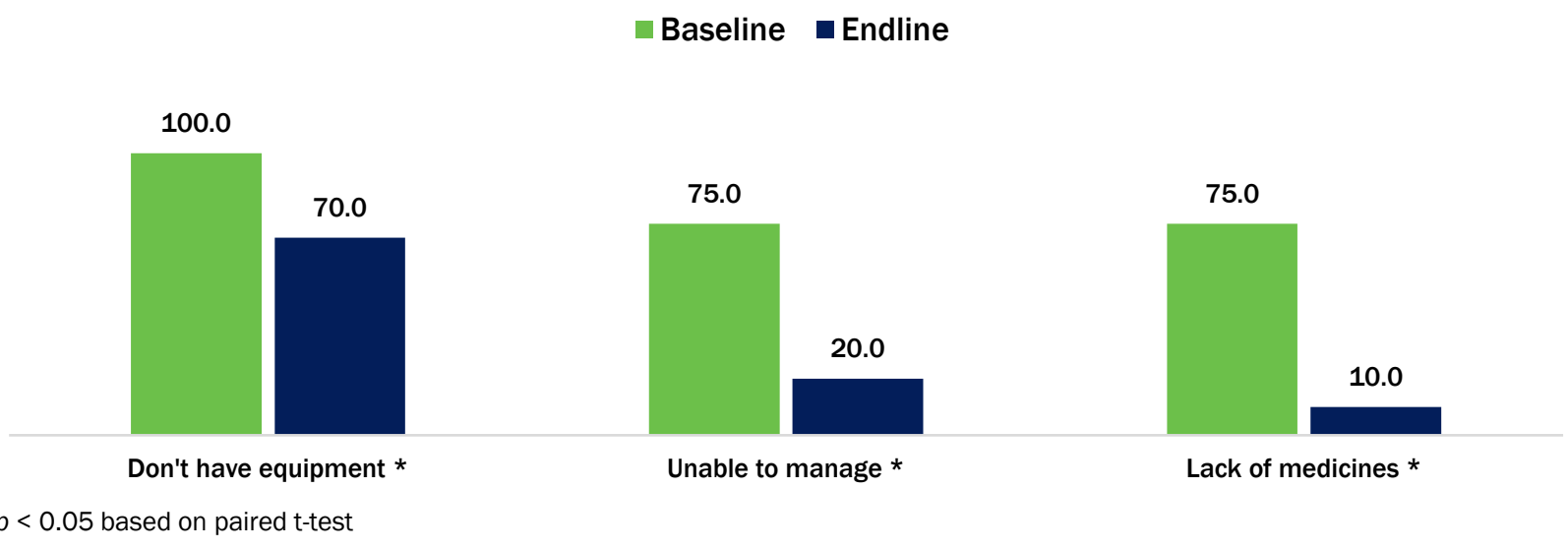

When asked for their additional thoughts or suggestions for improvement, more than half of CMWs recommended periodic refresher training (Figure 23). One tenth of CMWs thought the training should be of a longer duration, while less than one tenth believed it was sufficient in length. Another 10 percent of CMWs mentioned that the training was of good quality. A small proportion of respondents felt that training should guarantee job provision (3\%), that other community health workers should also be included (3\%), the referral system should be improved (3\%). Five percent of CMWs had no thoughts on the subject.

Figure 23: CMW opinions of training and suggestions for improvement, $n=37$

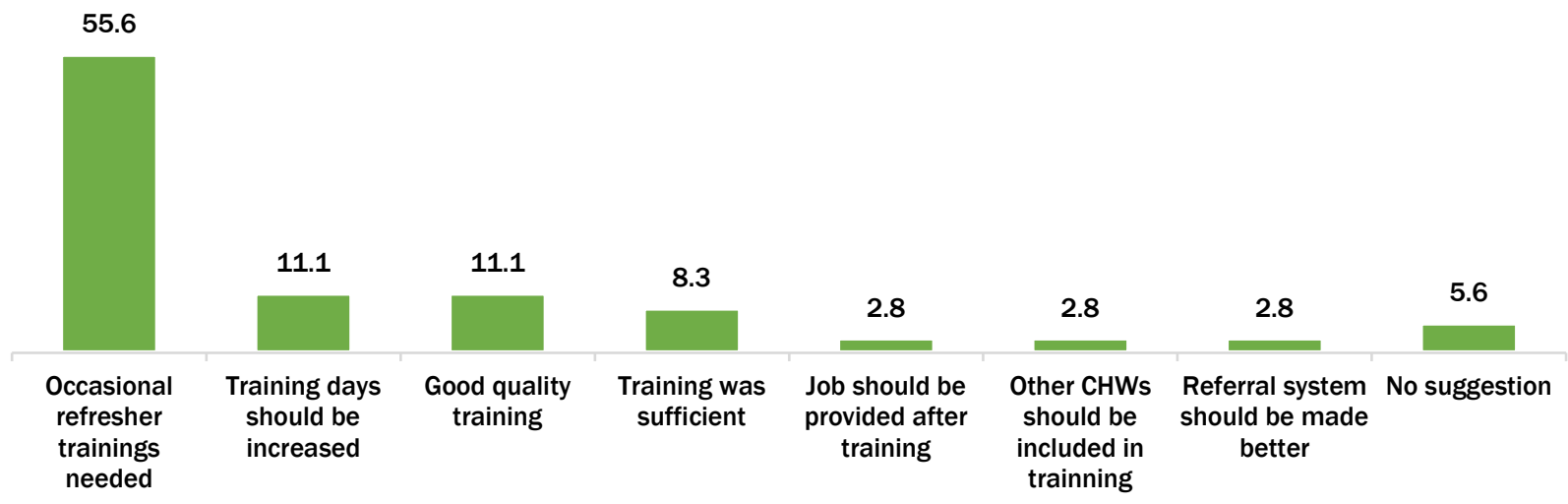




\section{Collaboration with Lady Health Workers}

In Arm Il's two tehsils, in Tando Allahyar and Chamber, LHWs were encouraged to refer pregnant women to CMWs and thereby hopefully improve women's access to MNCH services, in addition to developing sustained collaborative links with CMWs in their areas and communities. All CMWs, at both baseline and endline, knew all of the LHWs working in their area.

At endline, CMWs were asked about any coordination with local LHWs. Less than half of CMWs reported no coordination with a LHW in their area, while 41 percent said that they arranged group ANC meetings in coordination with a LHW (Figure 24). Overall, of the $37 \mathrm{CMWs,} \mathrm{five} \mathrm{reported} \mathrm{LHW} \mathrm{patient} \mathrm{referrals} \mathrm{to} \mathrm{them,}$ and four had jointly participated with local LHWs in monthly meetings at a health facility. During the intervention 89 group ANC sessions were conducted in total, on average four per month.

Figure 24: LHW coordination with CMWs reported by $\mathrm{CMWs}, \mathrm{n}=37$

\section{$\square$ Arm I $\square$ Arm II}

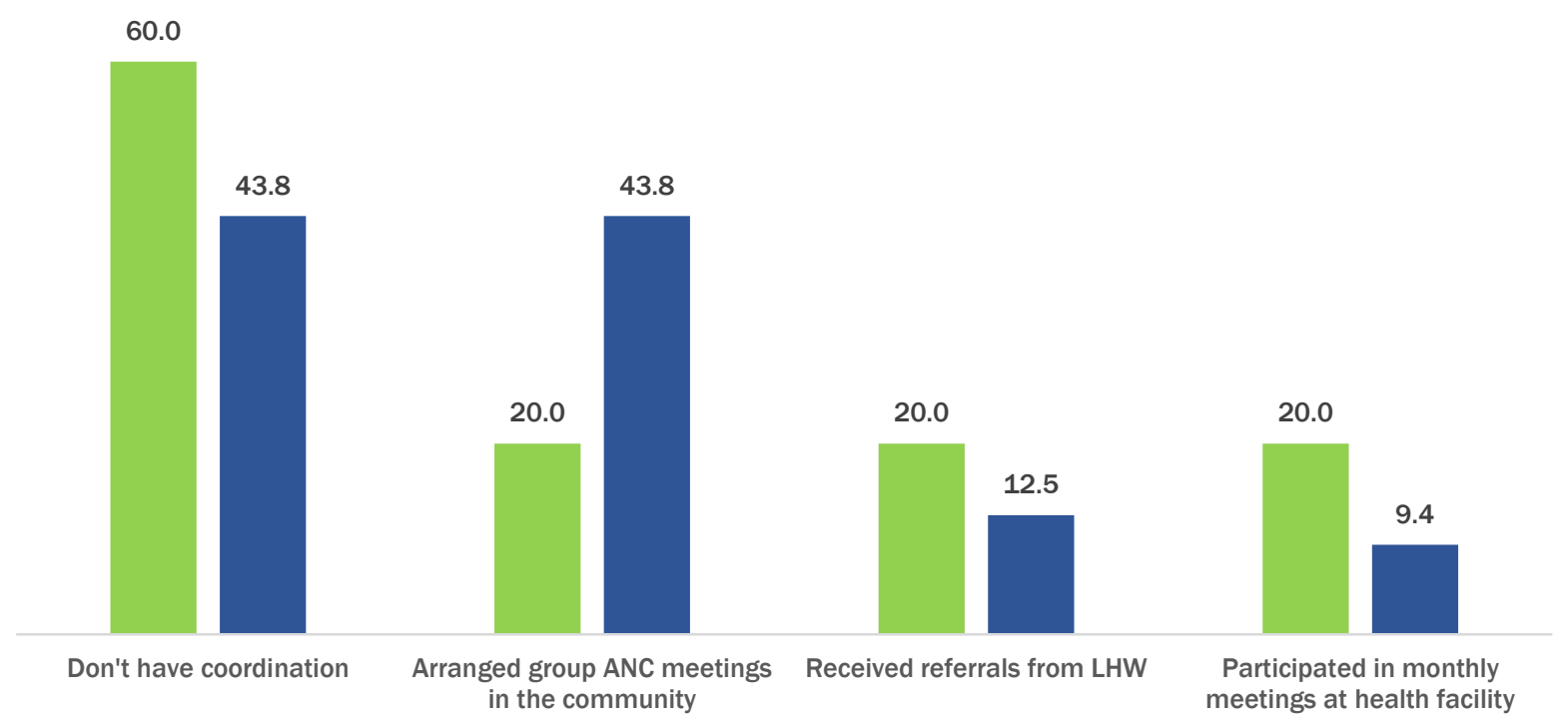

At endline, about three quarters of CMWs reported no professional links with a district health care worker in their community, for provision of ANC services to pregnant women, while approximately one quarter were linked with a LHW. Two CMWs were associated with a Lady Health Supervisor and Basic Health Unit staff person, respectively, and one with a NGO.

\section{Services Provided by Community Midwives, Based upon Service Statistical Data}

Antenatal care is preventive health care. Regular check ups allow CMWs to prevent potential health problems throughout the course of pregnancy in addition to promoting healthy lifestyles that will benefit both mother and child. The data presented in Figure 25 (following page) show, initially, fewer women approaching CMWs for ANC, but then the numbers gradually increase. Overall, 15,671 pregnant women received ANC from CMWs. Of those, 8,806 came to a CMW for the first time, and the remaining 6,865 were return visits, with an average of 33 patients per CMW per month during the intervention period (June 2017 through February 2019). 
Figure 25: ANC, delivery and PNC patients of CMWs during the intervention, $n=37$

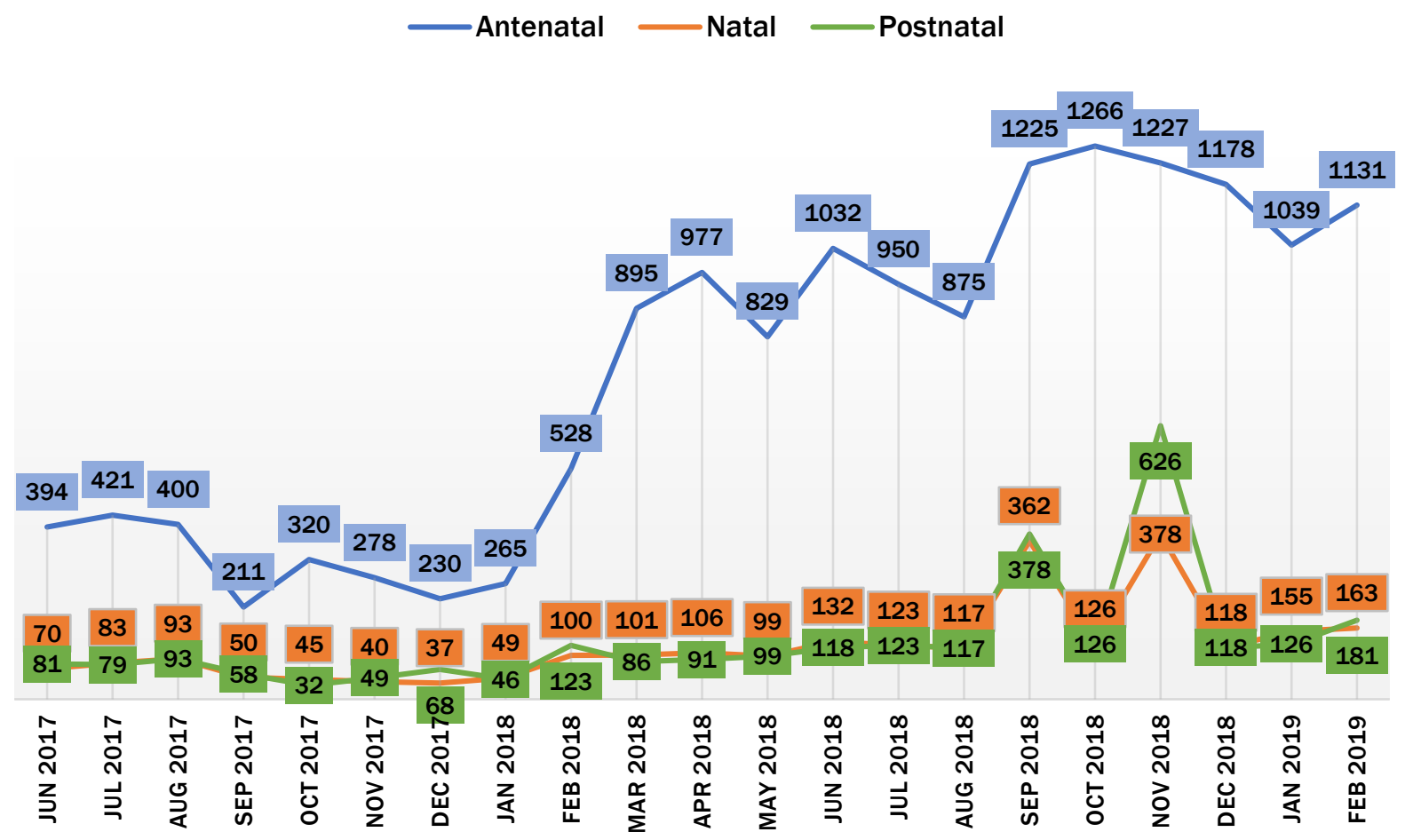

Overall, 2,547 deliveries were conducted by CMWs during the intervention period, out of which 2,229 were performed at their birthing stations, and 318 at client homes, within a cumulative average of five. A total of 2,818 women came to CMWs for PNC; the mean number was six per CMW per month.

During project implementation nine out of $37 \mathrm{CMWs}$ administered 39 loading doses of $\mathrm{MgSO}_{4}$.

Although not initially planned, Sindh's Director General of Health, at the second TAG meeting, recommended engaging private providers in the vicinity of Tando Allahyar so women in that peri-urban area could benefit from the intervention. Accordingly, private providers were trained in February 2018. From February 2018 to February 2019 private providers administered 77 loading doses.

Figure 26: $\mathrm{MgSO}_{4}$ doses by $\mathrm{CMWs}$ and private providers during the intervention, by month

CMWs Private providers

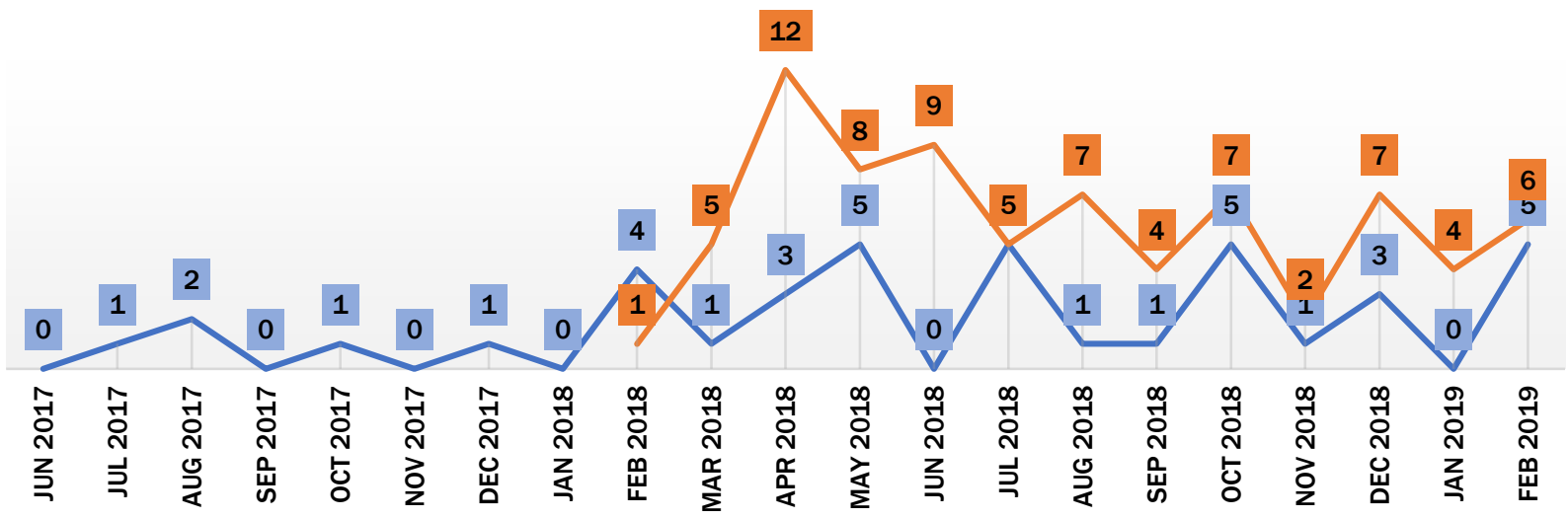




\section{Qualitative Findings}

\section{Women's Perceptions of Pregnancy Health Issues and the Roles of Community Midwives}

\section{Key Health Issues}

Women FGD informants reported high BP, anemia, and weakness as major problems during pregnancy. They identified lack of health facilities and unreliable supplies of medicines as the most common service delivery problems.

"High blood pressure is one of the major health issues for females...there is no facility to deal with emergencies. Sometimes blood loss causes complications at the time of delivery and no medicine is available at hospitals in our area. There is no laboratory for blood tests; if blood deficiencies are diagnosed early, then blood transfusion can be done on time, before getting into the kinds of complications that cause pregnant women to suffer from fits and die."

FGD, women, Chamber sub-district

"Unavailability of ultrasound and high blood pressure are issues that cause complications for pregnant women, due to which pregnant women can have fits and die."

FGD, women, Chamber

A few women also mentioned that postpartum hemorrhage is the most critical and potentially fatal condition after delivery, while the rest did not mention any possible complications after delivery.

"I have gone through an incident in my last pregnancy. I delivered at home, but after delivery I experienced postpartum hemorrhage and I fainted. My family immediately took me to hospital."

FGD, women, Missan village, Jhando Mari sub-district

\section{Awareness of Hypertension, Pre-Eclampsia and Eclampsia}

Women in Arm II who attended group ANC meetings with CMWs have better knowledge about PE/SPE/E. Women Jhando Mari sub-district (Arm I), with no ANC groups established, had limited PE/E knowledge; the only exception was a woman whose sister and niece had died due to eclampsia four years ago.

\section{Recognition of Dangers Associated with PE/E}

All women in Arm II (Tando Allahyar and Chambar sub-districts) where ANC groups were established, and who had participated in group ANC sessions, were aware of the side effects and potentially fatal outcomes of persistent high BP and PE/E during pregnancy.

"The community midwife gathered us at her clinic and provided us with the information. People [women] can be made aware in such ways too."

FGD, women, Tando Sumro village, Tando Allahyar

"All women said that now they are aware of the potentially fatal consequences of high blood pressure during pregnancy due to the awareness provided by the community midwife, who is functional and available in their respective areas. We attended a group ANC with our CMW. Such methods can be used for spreading awareness as well."

FGD, women, Tando Sumro village, Tando Allahyar

It was also suggested by most women (15 out of 26) in Arm II, who had gained knowledge from their CMWs about high $\mathrm{BP}$ during pregnancy and $\mathrm{PE} / \mathrm{E}$, that they should voluntarily visit pregnant women in the neighborhood to spread this knowledge. 
"We must visit pregnant women at home and must educate them regarding potential delivery complications at home. Then it's their own choice whether they follow our suggestion or not."

FGD, women, Chamber

The remaining 11 women said that CMWs can increase community awareness of pregnancy complications.

"Community midwife can provide us better information regarding pregnancy-related issues when she gathers us in form of antenatal care groups."

FGD, women, Tando Sumro village, Tando Allahyar

As ANC group meetings were not conducted in Arm I (Jhando Mari sub-district) those women were not fully aware of PE/E and its associated risks, yet all women in Jhando Mari said women must go to their CMWs so CMWs can provide all necessary information. All of these women in Arm I stated that LHWs can better spread PE/E awareness among community women because they are educated, have knowledge of pregnancy complications, and know who is currently pregnant, due to making home visits.

"The Lady Health Worker can make the pregnant women aware for dealing with and preventing pregnancy-related complications." FGD, women, Missan village, Jhando Mari

\section{Health-seeking Behaviors for Pregnancy Issues}

All women, from all three tehsils and study arms, said normal deliveries are usually conducted by CMWs, but for Caesarian section or other complications such as seizures, CMWs refer them to DHQ Tando Allahyar (or to the facility preferred by the client or her family) after providing first aid.

\section{Spiritual Treatment Versus Medical Care}

A majority of women in all three sub-districts prefer to visit medical professionals, either CMWs or doctors, for pregnancy issues, but a few mentioned belief in seeking both medical and "spiritual treatment." It is a cultural norm to visit to spiritual healers for their blessing in any time of difficulty. Spiritual treatment is also generally preferred by mothers-in-law. Women in the study areas generally lack formal education, with low levels of literacy, which are likely a factor in seeking redress with spiritual healers.

"The educated people, though, see high blood pressure as a medical problem and go to hospitals rather than any spiritual healer."

FGD, women, Tando Sumro village, Tando Allahyar

Two women from Chamber sub-district-of 26 total in Arm II-said that their in-laws wanted them to deliver at home and didn't allow them to go anywhere for pregnancy complications. Twenty-four of 26 women from Tando Allahyar and Chamber sub-districts said they went to their CMW for treatment of pregnancy complications, as she was closest, is professionally competent, and can deal with emergencies related to pregnancy.

"People must go to the nearest available community midwife for pregnancy-related complications."

FGD, women, Tando Sumro village, Tando Allahyar

All 26 women from Arm II (Tando Allahyar and Chamber) said eclampsia is a medical problem, and that women must go to doctors rather than spiritual healers.

"Well, the spiritual healers' procedure would only work for two hours or so and then the problem will start again, and he will have to start over [giggles]. All the time and money are wasted this way. Spending money on these spiritual healers leaves them without sufficient money to go to the hospital."

FGD, women Tando Sumro village, Tando Allahyar

"We always go to doctors rather than spiritual healers." GGD, women, Chamber 
Meanwhile, eight women-of 21-from Jhando Mari (Arm I) believe in spiritual treatment along with medical treatment. One woman even said she prefers spiritual treatment over medical treatment for pregnancy issues. The remaining 12 women said they go to hospitals for treatment of complications during pregnancy.

"We have to obey our mother-in-law as well and she believes that complications can be cured by the treatment [amulet] given by the spiritual healers, so we go to spiritual healers. But we want medical treatment, so we go to doctors as well." FGD, women, Jhando Mari

\section{Hypertension}

All 26 women in Arm II (Chamber and Tando Allahyar) said that they go to their CMWs whenever they feel elevated BP needing a check. In Jhando Mari (Arm I), 10 of 21 women said they go to doctors or hospitals for BP checks, while the other 11 said they went to their CMW for high BP during pregnancy.

"I come to the community midwife for antenatal check ups, and especially for postnatal check ups. She provides us with proper treatment; she administers an injection, and gives us medicine."

FGD, women, Missan village, Jhando Mari

\section{Household Decision-making}

Nineteen women-of 26 total-in Chamber and Tando Allahyar (Arm II) said their husbands are the family's major decision-maker, even for pregnancy health care, while six women said they participate, with the rest of the family, in care decisions. Only one woman, from Tando Allahyar, said she makes health care decisions for herself. In Jhando Mari (Arm I), 19 of 21 women said their family members or mothers-in-law make decisions about health care during pregnancy, while the remaining two women's husbands decide.

"Husband makes the decision where to seek health care during pregnancy."

FGD, women, Chamber

According to these women, informed families understand that high BP requires medication management, but if influential family members-mothers-in-law or husbands-are not informed, they perceive fits in pregnancy as the "work of some evil" and seek spiritual treatment, hoping for normal delivery at home.

"If the husband and mother-in-law are educated, they will go to doctors; if [they are] uneducated, they go to spiritual healers."

\section{FGD, women, Tando Sumro village, Tando Allahyar}

In most cases, parents-in-law and husbands are not well educated, which may be the reason for delayed care seeking for signs and symptoms of high BP that increase risk of maternal and infant mortality.

\section{Preference for Accessible and Affordable Sources}

Women generally seek treatment during pregnancy from CMWs because they are near and professionally qualified. All women in both study arms identified CMWs as educated health service providers who offer proper treatment without demanding a fixed fee. Sometimes CMWs not even charge for check ups, for BP or other general checks, if a woman cannot afford their services.

"Poverty is a big problem here, and most of the people here do not have enough money to get to the hospitals easily. Some of the doctors charge 300 Rupees, and some charge 400 Rupees. However, the community midwife does not ask for any money. She accepts as little an amount as 20 Rupees or sometimes 50 Rupees."

FGD, women, Tando Sumro village, Tando Allahyar

CMWs receive monthly stipend of 1,500 Rupees from the government, earning only nominal amounts from the communities they serve due to generalized local poverty. 


\section{Eclampsia}

Female FGD informants from all three tehsils are aware and appreciate the referral mechanism established by Ending Eclampsia allowing women who received a loading dose of $\mathrm{MgSO}_{4}$ from a $\mathrm{CMW}$ to then get a maintenance dose at a health facility. In the past, whenever visiting these facilities, women said they had not received adequate attention, but now, with the referral slip, they receive proper care.

"Hospital staff neither behave good nor provide services easily; they facilitate only when we are referred by community midwife."

FGD, women, Chamber

\section{Perceptions of Community Midwives}

According to the women interviewed, CMWs are always available, even late at night, and women from all three tehsils prefer going to their CMWs. Local women are satisfied with CMW care because they are trained to deal with emergencies. Another advantage is that the CMW accompanies the women to the referral hospitals whenever needed. A CMW does not demand any specific financial compensation but accepts whatever amount clients can give her. She even conducts check ups without charge.

"The community midwife lives near our homes in our neighborhood and she visits us at home. We can contact her at any time, even at late night; she is always available and never demands any fee."

FGD, women, Chamber

"The community midwife is available all the time, whether it's day or late night, and gives us proper treatment."

FGD, women, Missan village, Jhando Mari

"I come to the community midwife since she has started working here after receiving different trainings. She always cooperates with us."

FGD, women, Chamber

All community women expressed satisfaction with their treatment from CMWs and prefer CMW care to hospitals, as hospitals are farther away, requiring funds and transportation. CMWs are always present in their communities and provide proper treatment.

“We don't have hospitals in our area, and conveyance is another issue, when it comes to taking someone to the hospital in time."

FGD, women, Tando Sumro village, Tando Allahyar

"Previously, it was a preference to deliver at home, but now people are aware, and now we have a community midwife in our area, so we come here whenever we need."

FGD, women, Tando Sumro village, Tando Allahyar

"We come to the community midwife, even late at night, and she treats us properly. She is nearby."

FGD, women, Missan village, Jhando Mari

Apart from being affordable, easily accessible, and professionally competent, CMWs are also perceived as friendly, with most women in Tando Allahyar and Chamber sub-districts stating that CMWs' behavior is always nice-no matter what time they knock on their doors, they are always willing to help in every matter, even accompanying their patients to hospitals to prevent any kind of delay in treatment. Most women in Jhando Mari sub-district said that they get BP checks from CMWs for nominal fees, and at times without any fee, and that they sometimes visit their homes if they are unable to visit her.

"She is in our neighborhood and visits us at home. We can contact her at any time, even late at night. She is always available and never demands any fee." FGD, women, Chamber

"We always come to community midwife for antenatal care, delivery, or postnatal care, but if she refers us for delivery to hospital, we go..."

FGD, women, Jhando Mari 


\section{Experiences of Women Managed by Community Midwives for Pre-Eclampsia or Eclampsia}

Semi-structured interviews with survivors managed by CMWs for PE/SPE/E sought their opinions of their quality of care.

All 12 women said that money had been arranged in advance for delivery, and 11 women said their family was caring and transport was arranged. Concerning the services provided by their CMW or other providers, all the women affirmed the availability of first aid, availability of the CMW, competency of the service provider, good attitude of the provider, availability of medicine, and suitability of treatment.

When asked why they thought receiving ANC could help prevent complications, 11 out of the 12 women said that this contributed to early detection and management of any complications, prevention of maternal and infant death, as well as timely referral to a higher level health facility where needed.

The same number of women-11-said that during ANC their BP was measured, that they were informed of their pregnancy progress, were examined abdominally, and checked for anemia; 10 women reported that their infant's heartbeat was checked, and 10 also reported that their hands and feet were checked; nine women had a urine test and were told where to go if they noticed any danger signs, while eight were asked about their medical history; only six women were weighed.

Asked why they chose to approach a CMW for ANC, half of the women-six-said it was a family decision, and was of low cost, five said it was because the CMW lived nearby, two women chose a CMW because of her professional commitment, while one said no other option was available. All 12 women said that they would choose the same treatment if they suffered from high BP in their next pregnancy and would recommend the same treatment to anyone else having a similar condition.

When asked how they thought the problem of high BP or convulsions in pregnancy could be addressed, 10 women said timely treatment should be provided, nine responded that timely check ups are necessary, and three thought that better awareness is required and that information should be disseminated through LHWs, community teachers, and the media.

Eleven of the 12 women went to a CHW for PNC. Of these 11 women, five had one check up, three had two, and the other three had three check ups. During these check ups, nine women had their BP checked, eight were asked about their general well-being, seven were observed for bleeding, six were assessed for breastfeeding progress, three were checked for fever, and one woman was examined for abdominal pain.

When asked what they thought could happen to a pregnant woman with hypertension, all 12 women responded that 1) she could die, 2) there could be fetal death or a stillbirth, 3) both mother and child could die, 4) the woman could be sick even after delivery, and 5) the baby could born premature.

\section{Men's Perceptions of Women's Health Issues During Pregnancy}

\section{Most Common Health Issues in Pregnancy}

Men reported that the most common health problems faced by women during pregnancy included high blood pressure, anemia, and physical weakness. Twenty-five participants, out of 43 , reported high BP as a prominent health problem among pregnant women, while 18 men highlighted weakness during pregnancy as the most common problem that women face while pregnant. Most men said that expecting mothers are mostly anemic and weak due to malnutrition. 
“Weakness, anemia and high blood pressure is very common among pregnant women. A poor man who earns only 200 Rupees a day and has six to eight mouths to feed ultimately has limited financial resources to look after the nutrition and well-being of the family."

FGD, men, urban, Jhando Mari

\section{Pregnancy Care}

Thirteen of 43 male respondents mentioned that local CMWs are the major source of treatment for their wives during pregnancy. Eight out of 43 men said they took their wives to doctors and spiritual healers for treatment during pregnancies. All men prefer to take their wives to CMWs during pregnancy as well as at the time of delivery. They said CMWs are well-trained and competent, are in the near vicinity, and their attitudes towards their patients and their families are caring.

"We come to the community midwife here, and her behavior is very good. She explains everything very nicely. If she feels that there is need of a doctor, she refers us to them. She even helps us go to specific doctors by calling them in advance. She tells the doctor to treat her patient properly."

FGD, men, urban, Chamber

Every man said that he brings his wife to CMWs if they complain of any complication.

"We take our patient to the community midwife during the day or even at midnight. The community midwife comes to our home and treats very well. She gives either pills or injections as a treatment."

FGD, men, rural, Jhando Mari

\section{Knowledge of Hypertension, Pre-Eclampsia and Eclampsia and Their Care During Pregnancy and the Postnatal Period}

All 43 men did not evince much knowledge about pregnancy complications. Most did know, in cases of complications, that they should take their wives to the CMW, and if a problem is beyond the CMW's skills, she can refer them to the DHQ hospital or another nearby hospital. Going to the hospital also depends upon the will of family members and pregnant women themselves.

"We go to the community midwife and ask her opinion and do as she says. She refers us to the hospital in Tando Allahyar if it's not in her hands. This is what usually happens."

FGD, men, rural, Chamber

No male respondent could identify $\mathrm{MgSO}_{4}$ by name; they only knew that an injection is administered by CMWs. Men are less concerned with women's issues and do not accompany their wives during check ups with CMWs, as most of them are farmers and work in the fields.

"We are mostly at work but when we go back home then the wives tell us that they have been to a community midwife or to the doctor in Tando Allahyar."

FGD, men, rural, Jhando Mari

“My last child was also born with the community midwife's assistance. It's been 15 months since then. My wife had high blood pressure and she used to visit the community midwife. I don't know whether the community midwife gave her pills or injections. I never asked my wife."

FGD, men, rural, Jhando Mari

"The injections are given in cities but not here. Whatever medicines and facilities the community midwife have, she provides us, and if she doesn't have them, she prescribes for us to buy from the city."

FGD, men, rural, Jhando Mari 


\title{
Men's Perceptions of Community Midwives and Lady Health Workers
}

\section{Men's Perceptions of Community Midwives' Role in Health Services for Pregnant Women}

Almost all male respondents praised CMWs and expressed satisfaction with their performance and skills. They were also happy about the placement of CMWs in their area.

\begin{abstract}
"A community midwife deals with many health problems that a woman might face during pregnancy. She has most of the medicines but if she doesn't have them, she prescribes them and asks us to buy them from a medical store. A community midwife deals with blood pressure issues facing her patients and also takes care of deliveries. If a problem is more complicated, she refers the patient to the hospital." $\quad$ FGD, men, urban, Chamber

"It has been 15 years since my marriage, and I have never gone out for any medical help. I have always consulted the community midwife for any treatment that we need. My first child was born through a Cesarean operation and the rest of the children were delivered normally."

FGD, men, urban, Chamber

"She [CMW in the area] has been very helpful. Earlier, we used to take our patients to the hospital but then we came to know about the community midwife. She has been really helpful."

FGD, men, rural, Jhando Mari
\end{abstract}

\section{Lady Health Workers' Community Role}

Twenty-five male respondents-of 43-reported that LHWs are good sources of FP services. They also praised the LHWs for providing services at home and providing medicines to children and women. They expressed satisfaction with the quality of their services.

"The Lady Health Worker goes from door to door. She informs [clients] of birth spacing methods when she sees that the mother is getting weak. She provides family planning methods and provides treatment to women for birth spacing." FGD, men, urban, Chamber

\section{Lady Health Workers' Experiences}

\section{Collaboration with Community Midwives}

At endline, most LHWs in Arm II said that they had good working relationships with CHWs. As a result, pregnant women from their communities were delivered lectures by CMWs on pregnancy issues. The pregnant community women were linked by LHWs for these training sessions.

About half of (14 out of 27) LHWs in Arm II, where collaborative links were established between CMWs and LHWs, reported remaining with CMWs during infant deliveries. The remaining $13 \mathrm{LHWs}$ said that they only collaborate and organize ANC group meetings and PNC but are not present when CMWs conduct deliveries.

"Lady Health Workers are in contact at the time of child delivery with community midwives and provide all possible support to her." LHW, Kouro Daidano village, Arm II

In areas where the joint ANC intervention was not implemented (Arm I), LHWs report poor professional collaboration with CMWs. Some LHWs were less aware of CMWs' roles and responsibilities.

"Yes, there is a community midwife in our community, but people do not prefer them for deliveries. They only go to them for help in case of emergencies. Otherwise, they prefer to go to hospitals."

LHW, Pyaro Lund village, Arm I 
A few (4) LHWs from Arm I reported referring pregnant women for proper treatment as their routine job and responsibility to CMWs, but the majority only visit pregnant women during routine home visits.

"Although we are not present at the time of delivery, we visit them [women] within 24 hours [of their delivery] to check them and to guide them about their health because it is our primary duty to guide them."

LHW, Pyaro Lund village, Arm I

All 27 LHWs in Arm II said that they were officially informed regarding the deployed CMW in their areas and it was also reported that instructions were also given by district mangers to work in collaboration with each other because both are community health workers in their native communities.

All LHWs (26) from Arm I said CMWs do not refer any patient to them for vaccination or FP services, whereas 16 of 27 LHWs in Arm II, where ANC groups were formed, said CMWs send clients to them for vaccination and FP services; the remaining $11 \mathrm{LHWs}$ reported that CMWs do not refer any clients to them for FP or vaccination. On the other hand, 25 of $27 \mathrm{LHWs}$ in Arm II reported referring their clients to CMWs for ANC.

"When we are in the field then we follow up the women who are pregnant in the community and refer them to community midwives for antenatal check ups."

LHW, Kouro Daidano village, Arm II

"We refer patients [to the CMW] for delivery and if we doubt that the woman is pregnant, we refer her for blood pressure measurement and other checks."

LHW, Kouro Daidano village, Arm II

\section{Collaborative Group Antenatal Care and Reducing Risk of Unsafe Delivery and Related Diseases}

All LHWs in Arm II expressed satisfaction with group ANC sessions and observed that awareness among community women had increased after their participation. LHWs themselves felt more confident in referring patients to CMWs. LHWs feel that group ANC is the best forum for community women to receive and exchange knowledge that can save pregnant women's lives. Furthermore, community women's travel in groups to group ANC meetings is cost-effective.

Group ANC also proved to be good platforms for joint learning for community women, where they can share their own pregnancy-related problems with each other and obtain better advice.

"Community women will come to know the importance of antenatal checkups and rate of mortality will also decrease by attending group antenatal sessions, because pregnant women will come to know regarding health of mother and neonate, whether mother is anemic or not."

LHW, Kouro Didano village, Arm II

"When pregnant women come in groups, their travel cost is decreased."

\section{LHW, Kouro Didano village, Arm II}

"When women listen to other women's problem, they get knowledge that is new for them regarding pregnancy."

LHW, Kouro Didano village, Arm II

During the endline FGDs, 25 LHWs from Arm II reported sending groups of pregnant women to CMWs in different frequencies and gestational ages. 19 LHWs sent for group ANC of pregnant women of the same gestational age. In the baseline results, no action of this nature was reported.

Arm II, in which ANC group meetings were introduced, had comparatively better community service delivery by both provider cadres. 


\section{Discussion}

This Ending Eclampsia implementation research in Sindh's Tando Allahyar district was largely successful. Comparisons of baseline and endline data show improved CMW knowledge and performance in preventing, detecting, and managing PE/SPE/E, confirming the feasibility of interventions to improve community-based health care providers' knowledge and skills in areas of technical deficiency (provided interventions are properly planned and executed). Ending Eclampsia worked to improve CMWs' competencies by providing training that balanced theoretical concepts with application of newly acquired skills. In total, 116 women received a loading dose of $\mathrm{MgSO}_{4}$; whose lives were saved through prompt case management previously unavailable to them. During the intervention provision of $\mathrm{MNCH}$ services also increased, with a 17 percent increase in ANC clients, 10 percent more SBA deliveries, and nine percent more PNC patients seen.

Women recruited by female community health workers for this project are now acquainted with the health problems and risk factors of pregnancy, and are aware of the need for birth preparedness. Greater levels of awareness were seen in the two tehsils where the project worked with LHWs, and less so in the other tehsil. Communities appreciate CMWs' health care role because their services are affordable and, more importantly, are accessible and acceptable, as CMWs are part of the communities they serve, residing in the area. Women who were interviewed for this project had received a loading dose of $\mathrm{MgSO}_{4}$ and were satisfied, with no AEs reported. They all acknowledge that the injection saved their lives.

According to women in these communities, key decisions for critical health issues are made by their husbands. Therefore, enhancing men's knowledge of PE/SPE/E and the necessity of timely care is an important preventive strategy. Reliance on treatment from spiritual healers is generally advocated by senior members of families, such as mothers-in-law, which is a positive development.

Analysis of the baseline and endline data collected from CMWs, before and after the health care intervention, reflects marked improvement in CMWs' knowledge of the importance of ANC, PNC, along with maternal risk factors pre-disposing women to $\mathrm{PE} / \mathrm{SPE} / \mathrm{E}$, signs and symptoms of $\mathrm{PE} / \mathrm{E}$, and their management including urine albumin assessments $(p<0.05)$. The mean differences in knowledge between baseline and endline were statistically significant $(p<0.05)$.

To manage women with PE/SPE/E, a continuum of care is necessary, which required important structural changes. Tertiary care facilities in Hyderabad were included in the CMW referral system. Because a functional referral system is essential for prompt and timely case management, district referral facility staff were also included in PE/E trainings, which also enhanced their capacity and confidence. A major structural weakness was rectified by enabling DHQ hospital's treatment of eclamptic clients. The hospital's lack of a full time Ob/Gyn specialist and anesthetist prevented prompt Caesarian sections, an important element of effective PE/SPE/E management, and project efforts helped fill all vacant positions at the hospital, and emergency staff were fully briefed on referral protocols. The hospital also established a dedicated eclampsia room, with all necessary equipment and supplies for PE/SPE/E patients.

A major revelation and practical consideration was the need to include the private sector. Currently, 67 percent of deliveries in the district are facility-based, with 16 percent at public facilities and 51 percent at private clinics. It was clear the referral chain needed to be expanded to improve preventive services, by engaging private facilities to both provide PE/SPE/E services and act as referral points. Twenty private sector providers were engaged in PE/SPE/E management, an innovation that allowed urban and peri-urban women greater access to care. Of the total 116 women who received a loading dose of $\mathrm{MgSO}_{4}, 39$ were administered by CMWs, and 77 were from private providers. This project is an example of a promising public-private partnership, with private providers successfully managing cases, now part of a referral chain. 
Supportive supervision and improved monitoring are other key elements introduced by the project. Trained CMWs assembled each month at the $\mathrm{MNCH}$ office and submitted their monthly reports, discussed relevant issues, and received technical advice and support from district gynecologists, CMW instructors, and the MNCH director. Periodic visits to CMWs' birthing stations by project staff helped maintain standards.

Weaknesses are still seen in areas of less frequent home visits, along with persistent lack of equipment, and repair or replacement, as well as continued room for improvement in PNC knowledge. Coordination between CMWs and LHWs could also be further improved, but a promising start has been made.

The project worked with LHWs and CMWs in two tehsils to foster greater collaboration between the two cadres, engaging LHWs to refer their clients to CMWs for group ANC in addition to encouraging their promotion of PE/SPE/E awareness in their constituent communities. In total, 89 group ANC sessions were conducted by CHWs. On average, four such sessions were held per month. Meanwhile, there was a measurable increase in LHW presence during infant deliveries. Collaboration between the two cadres was not seen in the other tehsil of the district, where business continued as usual. Group ANC is appreciated by local women, as it is an opportunity for sharing experiences as well as group learning.

Major observations and lessons from this project's implementation countenance that CMWs need to have their knowledge and skills continuously updated along with regular supplies of medicines and essential equipment. The theoretical concepts learned by CMWs during their training need to be reinforced through practice, especially after deployment in communities. Periodic reinforcement and refresher training, along with practical support, will maintain the utility and vitality of this cadre of providers. A 2016 study in Sindh found several gaps in knowledge of pre-eclampsia etiology, diagnosis, and treatment among health care providers-including both facility and community-based providers ${ }^{15}$. Essential medicines and supplies should be provided through a formal mechanism, as CMWs alone cannot be expected to both purchase these supplies and provide affordable care.

Systematic and programmatic challenges were observed. CMW attrition is a matter of concern. Attracted by more lucrative opportunities, some CMWs leave their communities to work in urban facilities or seek employment from PPHI. CMWs also do not receive their stipends, mandated for two years, regularly. Their health houses or birthing stations are also not fully to standard and require refurbishment and improved hygiene practices.

The project worked with Sindh's DoH from the outset, and through the provincial and district TAGs comprised of government officials and $\mathrm{NCMNCH}$ president and members, ensuring important institutional support for project activities along with systemic integration. The intervention's positive results are appreciated by Sindh's DoH (Appendix 4) and the TAG. Sindh's Director General of Health recommended the project's scale up to the province's other 29 districts. Accordingly, seven one day orientation sessions were organized for 63 district managers including District Health Officers and District Coordinators of the LHW and $\mathrm{MNCH}$ programs on replicating the project's interventions. In addition, 45 referral facility gynecologists and WMOs were oriented on the referral system and how to support CMWs' eclampsia management. Furthermore, $43 \mathrm{CMW}$ midwifery school instructors and principals were oriented on the practical aspects of the competency-based trainings. District managers have committed to working with DoH for procuring adequate $\mathrm{MgSO}_{4}$ stocks to ensure regular CMW supply, and gynecologists agreed to technical supervision. Midwifery school staff will employ the training materials and focus on further disseminating the important lessons from this project's implementation.

${ }^{15}$ Sheikh S1 ${ }^{1}$ RN Qureshi², AR Khowaja ${ }^{1,3}$, R Salam ${ }^{1}$, M Vidler $^{3}$, D Sawchuck ${ }^{4}$, P von Dadelszen ${ }^{5}$, S Zaidi $^{1}$, Z Bhutta1,6, CLIP Working Group. Health care provider knowledge and routine management of pre-eclampsia in Pakistan. 


\section{Conclusion}

Our results reaffirm that simple interventions, if implemented effectively, can yield positive results-but successful effortts must be integrated and scaled up so their benefits are enjoyed by the entirety of a population.

\section{Recommendations}

- All CMWs establishing functional birthing stations must be regulated, supervised, and supported by the health system. The MNCH program's CMW selection criteria should be thorough, with diligent area mapping.

- Competency-based skill development training must be provided to all CMWs, as well as facility-based providers, to enhance their capacities and confidence in administering a loading dose of $\mathrm{MgSO}_{4}$ and referring those clients to appropriate facilities for a maintenance dose.

- Stronger monitoring, response, and referral mechanisms must be incorporated within the CMW program to ensure quality services for community women.

- The referral system must be strengthened to include CMWs in the referral course so their referred clients are guaranteed appropriate and timely care.

- $\mathrm{PE} / \mathrm{E}$ management protocols, especially for $\mathrm{MgSO}_{4}$ administration, should be widely distributed to ensure universally availability, with prominent displays at all health facilities.

- Careful follow up with providers is crucial for reinforcing knowledge and skills at their actual locations of care provision, enabling CMWs to more confidently deal with potentially life-threatening conditions.

- With 32 percent of deliveries still outside the health system, at home, investing in CMW skills improvements, to manage the three most important causes of maternal mortality-postpartum hemorrhage, sepsis, and eclampsia-is a vital pre-requisite for lowering Pakistan's MMR. Community interventions for strengthening the skills and competencies of CMWs, to deal with these three emergencies, should be a priority.

- Collaboration between the LHW and CMW programs can be strengthened if the MNCH and LHW programs work synergistically and cooperatively, with formal mechanisms established for LHW referrals of pregnant women to local CMWs for ANC, delivery care, and PNC.

- CMWs' birthing stations need renovation and critical features such as running water and washrooms, among others. Basic equipment is frequently missing or in disrepair, such as BP apparatuses, which were provided by the project. This deficiency of essential supplies needs to be corrected. 


\section{Appendices}

\section{Appendix 1: Availability of Drugs and Contraceptives}

Among Community Midwives

Number of community midwives by availability of medicines

\begin{tabular}{|l|c|c|c|}
\hline \multicolumn{1}{|c|}{ Type of medicines } & Baseline & Endline & Change \\
\hline Micronutrients Tablets & 30 & 37 & $\uparrow$ \\
\hline Antibiotics Injectable Oral & 32 & 37 & $\uparrow$ \\
\hline Analgesics Injectable Oral & 32 & 37 & $\uparrow$ \\
\hline Antiseptics & 31 & 37 & $\uparrow$ \\
\hline Methergine & 33 & 37 & $\uparrow$ \\
\hline Antifungal Vaginal Tablets with applicator & 37 & 37 & - \\
\hline MgSO injection & 34 & 37 & $\uparrow$ \\
\hline Aspirin tablet & 37 & 37 & - \\
\hline Misoprostol tablet & 31 & 37 & $\uparrow$ \\
\hline Aldomet & 32 & 37 & $\uparrow$ \\
\hline Nifidepine & 36 & 37 & $\uparrow$ \\
\hline Hydralazine & 36 & 36 & - \\
\hline Disposable syringe 10cc & 34 & 35 & $\uparrow$ \\
\hline Disposable syringe 20cc & 34 & 35 & $\uparrow$ \\
\hline Ringers Solution & 33 & 35 & $\uparrow$ \\
\hline Saline I/V drip & 32 & 35 & $\uparrow$ \\
\hline Dextrose I/V drip & 34 & 36 & $\uparrow$ \\
\hline
\end{tabular}

Number of community midwives by availability of contraceptives

\begin{tabular}{|l|c|c|c|}
\hline \multicolumn{1}{|c|}{ Type of contraceptives } & Baseline & Endline & Change \\
\hline Pills & 30 & 36 & $\uparrow$ \\
\hline Condoms & 29 & 36 & $\uparrow$ \\
\hline Injectables & 30 & 36 & $\uparrow$ \\
\hline Intrauterine Contraceptive Devices (IUCDs) & 2 & 14 & $\uparrow$ \\
\hline ECPs & 0 & 6 & $\uparrow$ \\
\hline
\end{tabular}




\section{Appendix 2: Ethical Approval}

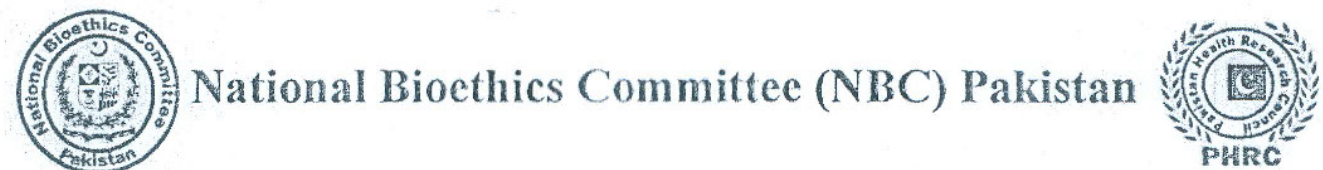

Ref: No.4-87/17/NBC-229/NBC/3/23

Date: $14^{\text {th }}$ March, 2017

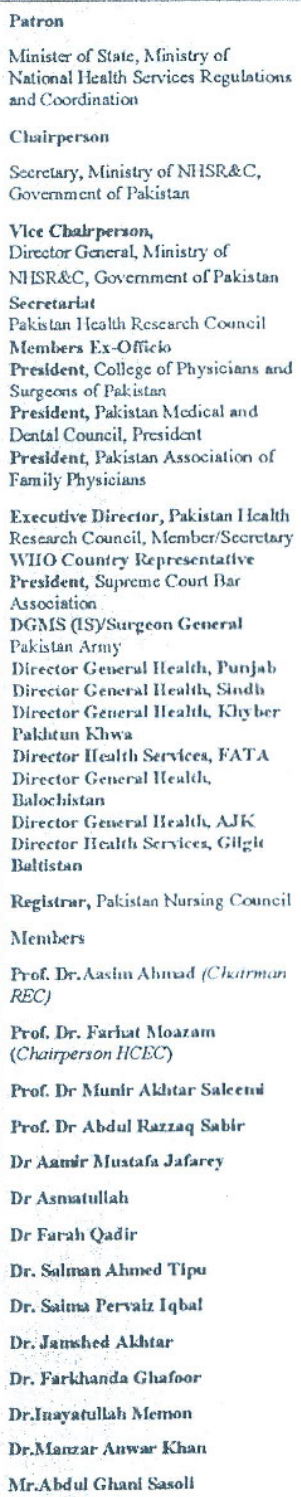

Dear Dr. Ali Mohammad Mir,

I am pleased to inform you that the above mentioned project has been cleared by the "Research Ethics Committee" of "National Bioethics Committee" for a period of one year.

For the continuation of project in the next years, you have to send a progress report and a formal request asking for continuation of projects (however, you do not need to submit REC application or pay any processing fee again).

Kindly keep the National Biocthics Committee, Secretariat updated about the progress of the project and submit the formal final report on completion.

Yours sincerely

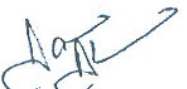

(Prof. Dr. Aasim Ahmad)

Chairman

NBC- Research Ethics Committee

Pakistan Health Research Council, Shahrah-e-Jamhuriat, Off Constitution Avenue, Sector G-5/2, Islamabad www.nbepakistan org.pk, e-mail: nbenakistan orgagmail.com Tel: 92-51-9224325, 9216793, Fax 9216774 


\title{
Appendix 3: Technical Advisory Group
}

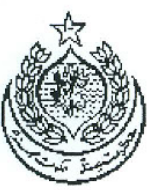

\author{
DIRECTORATE GENERAL HEALTH SERVICES \\ SINDH@HYDERABAD
}

Phone \# 022-9240106 Fax \# 022-9240100

E-mail:dghealthsindh@sindhealth.pk

No. DGHSS/ - (P.H-Wing-Pop: Council)/-1845/61 /Dated gg - $-03-2017$
To,

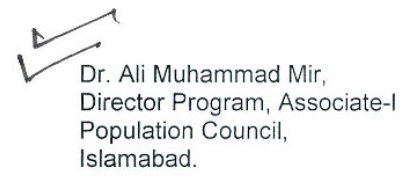

Subject:

TECHNICAL ADVISORY GROUP FOR FIVE YEAR PROJECT ENTITLED "ENDING ECLAMPSIA".

Reference:- $\quad$ Letter No. Nil dated 28-02-2017 received from Director Program, Associate-I, Population Council, Islamabad.

With refer to the letter quoted above, as per decision of inception meeting held on 16-02-2017 at Karachi following are notified as member of Technical Advisory Group (TAG) for Five year Project Entitled "Ending Eclampsia"

\begin{tabular}{|c|c|c|}
\hline 1. & $\begin{array}{l}\text { Dr. Muhammad Tofique, Director General Health Services Sindh } \\
\text { at Hyderabad. }\end{array}$ & Chairman \\
\hline 2. & Dr. Shahib Jan Badar, Program Manager, MNCH Sindh & Co-Chair \\
\hline 3. & $\begin{array}{l}\text { Dr. Muhammad Saleem Memon, Additional Director(PH) at } \\
\text { Directorate General Health Services Sindh at Hyderabad }\end{array}$ & Member \\
\hline 4 & $\begin{array}{l}\text { Dr. Ghazala Hamirani, Additional Director }(\mathrm{RCH}) \text { at Directorate } \\
\text { General Health Services Sindh at Hyderabad. }\end{array}$ & Member \\
\hline 5 & $\begin{array}{l}\text { Provincial Coordinator, National Program for Family Planning } \\
\text { and PHC Hyderabad. }\end{array}$ & Member \\
\hline 6 & Dr. Shabir Chandio, Health Management Specialist USAID. & Member \\
\hline 7 & Dr. Iftikhar Malah, Deputy Project Director $\mathrm{MNCH}$ & Member \\
\hline 8 & Dr. Sadiqua Jaffray, President NCMNH. & Member \\
\hline 9 & Dr. Azra Hassan, Technical Expert NCMNH & Member \\
\hline 10 & Dr. Ali Mir, Principal, Investigator. & Member \\
\hline 11 & Dr. Rashida Gul. Technical Advisor Population Council. & Member \\
\hline 12 & Dr. Saleem Shaikh, Study Manager & Coordinator \\
\hline
\end{tabular}

$$
\text { DIRECTOR GENERAL }
$$

HEALTH SERVICES SINDH AT HYDERABAD

CC.

- The Secretary, Government of Sindh, Health Department Karachi.

- P.S. to Minister for Health, Government of Sindh, Health Department Karachi.

- The Program Manager, MNCH Sindh, Karachi.

- The Provincial Coordinator, National Program for Family Planning and PHC Hyderabad

- The Additional Director (PH), Directorate General Health Services Sindh at Hyderabad.

- The Additional Director RCH, Directorate General Health Services Sindh at Hyderabad.

- All Members of Technical Advisory Group. 


\title{
Appendix 4: Letters of Official Agreement
}

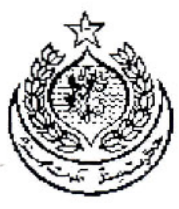

\author{
DIRECTORATE GENERAL HEALTH SERVICES \\ SINDH@HYDERABAD \\ Phone \#022-9240106 Fax \#022-9240100 \\ E-mail: dghealthsindh@sindhealth.pk
}

\begin{abstract}
No. DGHSS/ - (P.H-Wing-Pop: Council)/- ///3//7 IDated $16-02-2017$
To.
$>$ The District Health Officer, Tando Allahyar.

$>$ The Provincial Program Director, National MNCH Program Sindh Karachi.

The Provincial Coordinator, national Program for Family Planning and PHC Hyderabad.
\end{abstract}

Subject: $\quad$ QUASI-EXPERIMENTAL PRETEST-POST-TEST STUDY.

A copy of letter dated 09-02-2017 received from Associate I/ Director Programs, Population Council Islamabad is forwarded herewith with the advise to extend the full cooperation to the Population Council's staff to conduct this study under intimation to this office.

Encl: As above

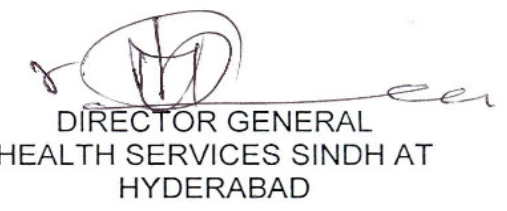

CC:

The Secretary, Government of Sindh, Health Department Karachi.

> The Associate l'Director Programs, Population Council Islamabad.

E:P. Council.docx 


\section{OFFICE OF THE DISTRICT HEALTH OFFICE TANDO ALLAHYAR}

NO: DHO / TAR (MNCH) /-

Dated 12017.

To,

Mr Ali Muhammad Mir,

Director Program Population Council Islamabad.

Subject

\section{SUPPORT LETTER}

With reference to letter NO: DGHSS / (P.H-Wing-Pop: Council) /- 1113/17

Dated $16^{\text {th }}$ February 2017, District Health Department will be provide full support in the implementation of project activities and coordination's

Ce to:

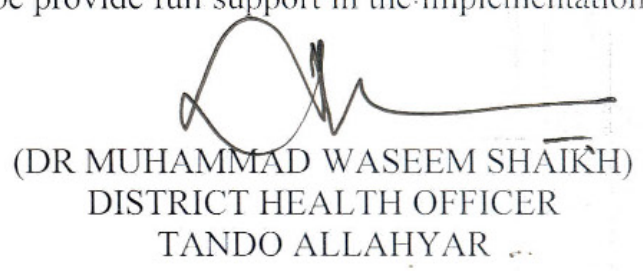

1. The Director General Health Services Sindh Hyderabad.

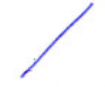

(DR MUHAMMAD WASEEM SHAIKH)

DISTRICT HEALTH OFFICER

TANDO ALLAHYAR

w/documents/desktop file gnabi-2016 CMWSTAR 


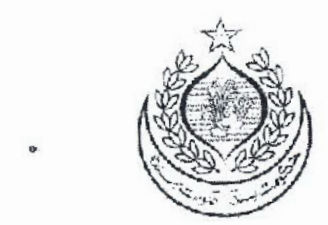

NO.DGHSS/HR-I/ (PF-)/ S $/ 11 / 14$

To,

The District Health Officer

Tando Allahyar.

\section{SUBJECT: APPRECIATE.}

I appreciate your co-operation regarding "Expanding Services to Detect,
DIRECTORATE GENERAL

HEALTH SERVICES SINDH @ HYDERABAD

Phone \# 022-9240106 FAX \# 0229240100

(email; dghealthsindh@sindhealth.pk)

\section{SUBJECT: APPRECIATE.}

Dated:20-ol- 2018.

Prevent severe Pre-eclampsia / Eclampsia in Pakistan" in District Tando Allahyar.

I wish you devoted efforts for the improvement of your District and more successes in future.

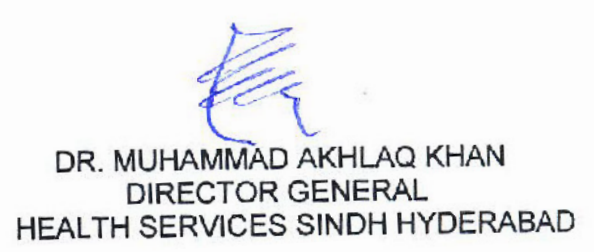

CC:

- The Secretary Government of Sindh Health Department Karachi.

- The P.S to Minister Health Sindh Karachi.

- Dr. Ali Muhammad Mir Associate / Director Programs Population Council Ideas, Evidence, Impact, $3^{\text {rd }}$ Floor NTC Building (North) Sector F-5/1 Islamabad. 